MATHEMATICS OF COMPUTATION

Volume 74, Number 251, Pages 1495-1518

S 0025-5718(05)01730-8

Article electronically published on January 27, 2005

\title{
COMPUTING THE TIGHT CLOSURE IN DIMENSION TWO
}

\author{
HOLGER BRENNER
}

\begin{abstract}
We study computational aspects of the tight closure of a homogeneous primary ideal in a two-dimensional normal standard-graded domain. We show how to use slope criteria for the sheaf of relations for generators of the ideal to compute its tight closure. In particular, our method gives an algorithm to compute the tight closure of three elements under the condition that we are able to compute the Harder-Narasimhan filtration. We apply this to the computation of $\left(x^{a}, y^{a}, z^{a}\right)^{*}$ in $K[x, y, z] /(F)$, where $F$ is a homogeneous polynomial.
\end{abstract}

\section{INTRODUCTION}

Let $I \subseteq R$ denote an ideal in a Noetherian domain $R$ over a field $K$ of characteristic $p>0$. The tight closure of $I=\left(f_{1}, \ldots, f_{n}\right)$ is again an ideal defined by

$$
I^{*}=\left\{f \in R: \exists c \neq 0: c f^{q} \in\left(f_{1}^{q}, \ldots, f_{n}^{q}\right) \text { holds for almost all } q=p^{e}\right\} .
$$

The theory of tight closure was developed by Hochster and Huneke (see [10, [11, [12], 22]) and has many applications in commutative algebra, homological algebra and algebraic geometry. Its strength lies in the interplay of inclusion and exclusion results for tight closure.

Huneke writes, "Tight closure is very difficult to compute; indeed that is necessarily the case. It contains a great deal of information concerning subtle properties of the ring and the ideal" ([11, Basic Notions]). The problem lies in the fact that due to the definition we have to check infinitely many conditions.

If the ring $R$ is regular, then $I=I^{*}$ holds for every ideal $I \subseteq R$. If the ring is one-dimensional, then $I^{*}=R \cap I R^{\text {nor }}$ (the normalization), so in these two cases the computation of $I^{*}$ is easy (at least there is a translation to other more elementary computational problems). But even in the case of a normal twodimensional standard-graded domain $R$ over an algebraically closed field $K$ (e.g., $R=K[x, y, z] /(F), F$ homogeneous) very little is known. The tight closure of a homogeneous parameter ideal $\left(f_{1}, f_{2}\right) \subset R$ is given by

$$
\left(f_{1}, f_{2}\right)^{*}=\left(f_{1}, f_{2}\right)+R_{\geq \operatorname{deg}\left(f_{1}\right)+\operatorname{deg}\left(f_{2}\right)}
$$

(in characteristic 0 or $p \gg 0$ ). This is the Strong Vanishing Theorem of Huneke and Smith ([13]), which has been generalized for parameter ideals in higher dimensions by Hara $([5])$.

Received by the editor March 10, 2003 and, in revised form, April 11, 2004.

2000 Mathematics Subject Classification. Primary 13A35; Secondary 14H60.

(C)2005 American Mathematical Society 
For a homogeneous $R_{+}$-primary ideal $I=\left(f_{1}, \ldots, f_{n}\right) \subseteq R$ not much is known about the tight closure $\left(f_{1}, \ldots, f_{n}\right)^{*}$ for $n \geq 3$. A difficult but elementary computation due to Singh shows that $x y z \in\left(x^{2}, y^{2}, z^{2}\right)^{*}$ holds in the Fermat cubic given by $x^{3}+y^{3}+z^{3}=0([20]$, [16]). Smith has given the two degree bounds

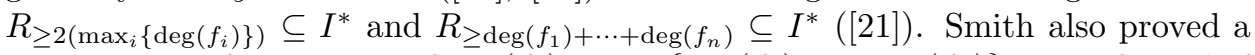
degree bound from below: if $\operatorname{deg}\left(f_{0}\right) \leq \min \left\{\operatorname{deg}\left(f_{1}\right), \ldots, \operatorname{deg}\left(f_{n}\right)\right\}$, then $f_{0} \in I^{*}$ if and only if $f_{0} \in I$ already. All these bounds are rather coarse for nonparameter ideals. If the $f_{i}$ have the same degree $d$, then these degree bounds say nothing between $d$ and $2 d$. In particular they do not yield anything interesting in the example of Singh.

Another approach was initiated by Katzman and further developed by Sullivant. They use an algorithm which computes, given an ideal $I$, two ideals $I_{1}$ and $I_{2}$ such that $I_{1} \subseteq I^{*} \subseteq I_{2}$. If both approximations coincide, then the algorithm gives the right answer for a fixed prime number $p$. The computations of Sullivant (implemented in Macaulay2) of $\left(x^{3}, y^{3}, z^{3}\right)^{*}$ for the Fermat rings $K[x, y, z] /\left(x^{d}+y^{d}+z^{d}\right)$ for $p \leq 53$ and $d \leq 26$ are striking and have led to some interesting observations and conjectures. Of course one cannot expect any general result by this method, and the lower bound $I_{1}$ computes rather the Frobenius closure of the ideal. Therefore the algorithm does not give the right answer for $\left(x^{2}, y^{2}, z^{2}\right)^{*}$ for $p=1 \bmod 3$.

In this paper we want to attack the problem of computing the tight closure of an ideal from another point of view: using the slope criteria for vector bundles. This rests upon the geometric interpretation of tight closure via vector bundles and projective bundles which we have developed in [1] and [2]. This paper will emphasize the computational usefulness of this approach.

The main object to consider in this approach is the sheaf of relations of total degree $m$ for homogeneous ideal generators $f_{1}, \ldots, f_{n}$. This is a locally free sheaf $\mathcal{R}(m)$ on the smooth projective curve $Y=\operatorname{Proj} R$. The slope properties of this sheaf are crucial for the underlying tight closure problem. So we may forget the definition of tight closure and struggle instead with the notions of slope, minimal and maximal slope, semistability and the Harder-Narasimhan filtration of this sheaf of relations. This is still a difficult task, however we can use many more tools from algebraic geometry to attack the tight closure problem. We recall this geometric interpretation and the resulting slope criteria for tight closure briefly in Section 1 .

In fact we work with the notion of solid closure (denoted $I^{\star}$ ), which coincides with tight closure in positive characteristic and gives a satisfactory notion for characteristic zero in dimension two. The slope conditions are easier to formulate in zero characteristic; hence we restrict largely to this case in the introduction.

If the sheaf of relations $\mathcal{R}(m)$ is semistable, then we have an easy numerical criterion for tight closure: the common degree bound for inclusion and exclusion is given by $\frac{\operatorname{deg}\left(f_{1}\right)+\cdots+\operatorname{deg}\left(f_{n}\right)}{n-1}$. If the sheaf of relations is not semistable, then we can argue along the Harder-Narasimhan filtration of $\mathcal{R}(m)$. This yields the first step of an algorithm to compute the tight closure. This algorithm gives a complete answer if the maximal destabilizing subsheaf of the sheaf of relations is itself semistable. This condition is of course fulfilled if the rank of $\mathcal{R}(m)$ is two; hence we get an algorithm to compute the tight closure of a homogeneous $R_{+}$-primary ideal generated by three Elements (Section 2) - at least if we are able to compute the Harder-Narasimhan filtration, which means for rank two to find the invertible subsheaves of maximal 
degree (equivalently, to compute the $e$-invariant of the corresponding ruled surface). This method works also in positive characteristic and we get (less complete) results about the plus closure (Section [3).

In Section 4 we study the global sections of the sheaf of relations for homogeneous ideal generators. Their existence and nonexistence in certain degrees has many consequences on the slope properties of $\mathcal{R}(m)$ and therefore on the tight closure. For example, if there does not exist a relation $\neq 0$ of total degree $k$ for the homogeneous primary elements $f_{1}, f_{2}, f_{3}$, then

$$
R_{m} \subseteq\left(f_{1}, f_{2}, f_{3}\right)^{\star}
$$

holds for

$$
m \geq d_{1}+d_{2}+d_{3}-k+\frac{g-1}{\delta},
$$

where $g$ denotes the genus and $\delta$ the degree of $Y$ (Corollary 4.4). The nonexistence of global relations also implies exclusion results: if there does not exist a global nontrivial relation for $f_{1}, \ldots, f_{n} \in R=K[x, y, z] /(F)$ of degree $k$, then

$$
\left(f_{1}, \ldots, f_{n}\right)^{\star} \cap R_{m}=\left(f_{1}, \ldots, f_{n}\right) \cap R_{m}
$$

holds for $m \leq k-\delta+2$, where $\delta$ is the degree of $F$ (Proposition 4.10).

On the other hand, the existence of global relations also yields results about the tight closure (Section 5). If there exists a primary relation (that is, a relation such that the quotient is locally free) for $f_{1}, f_{2}, f_{3}$ of total degree $k \leq\left(d_{1}+d_{2}+d_{3}\right) / 2$, then $f_{0} \in\left(f_{1}, f_{2}, f_{3}\right)^{\star}$ holds for $m \geq d_{1}+d_{2}+d_{3}-k$ and $f_{0} \in\left(f_{1}, f_{2}, f_{3}\right)^{\star}$ if and only if $f_{0} \in\left(f_{1}, f_{2}, f_{3}\right)$ for $m<k$ (Corollary 5.3).

In Section [6] we consider the tight closure $\left(x^{a}, y^{a}, z^{a}\right)^{\star}$ in $R=K[x, y, z] /(F)$, where $F$ is a homogeneous polynomial of degree $\delta$ defining a smooth projective curve. Our main result is that

$$
\left(x^{a}, y^{a}, z^{a}\right)^{\star}=\left(x^{a}, y^{a}, z^{a}\right)+R_{\geq \frac{3}{2} a}
$$

holds for

$$
\delta \geq 3 a-1
$$

(Corollary 6.3) in characteristic zero. In positive characteristic we show that

$$
R_{m} \subset\left(x^{a}, y^{a}, z^{a}\right)^{\star}
$$

(and even in the Frobenius closure) holds for

and that

$$
m>\frac{3}{2} a, \quad \delta \geq 3 a-1, \quad p>\delta-3
$$

holds for

$$
R_{m} \cap\left(x^{a}, y^{a}, z^{a}\right)^{\star}=R_{m} \cap\left(x^{a}, y^{a}, z^{a}\right)
$$

$$
m<\frac{3}{2} a, \quad \delta \geq 3 a-1, \quad p \gg 0
$$

(Corollary 6.5)).

In Section[7] we have a closer look at $\left(x^{2}, y^{2}, z^{2}\right)^{\star}$ and $\left(x^{3}, y^{3}, z^{3}\right)^{\star}$ in $K[x, y, z] /(F)$ for $F$ of low degree $\delta$. We extend some of the general results to degree $\delta<3 a-1$ and we prove some of the conjectures of Sullivant to which he was led by his computations of $\left(x^{3}, y^{3}, z^{3}\right)^{\star}$ in the Fermat rings.

I would like to thank the referee for careful reading and useful remarks. 


\section{Slope CRIteria FOR tight Closure}

We recall the main results of [2]. Let $R$ denote a normal two-dimensional standard-graded $K$-domain over an algebraically closed field $K$ and let $f_{1}, \ldots, f_{n}$ denote homogeneous $R_{+}$-primary elements of degree $d_{i}=\operatorname{deg}\left(f_{i}\right)$. These elements define the locally free sheaf of relations $\mathcal{R}(m)$ on the smooth projective curve $Y=\operatorname{Proj} R$ given by the short exact sequence

$$
0 \longrightarrow \mathcal{R}(m) \longrightarrow \bigoplus_{i=1}^{n} \varnothing_{Y}\left(m-d_{i}\right) \stackrel{f_{1}, \ldots, f_{n}}{\longrightarrow} \emptyset_{Y}(m) \longrightarrow 0
$$

Another homogeneous element $f_{0}$ of degree $m$ yields via the connecting homomorphism a cohomology class $c=\delta\left(f_{0}\right) \in H^{1}(Y, \mathcal{R}(m))$. This class corresponds to an extension $(\operatorname{set} \mathcal{S}=\mathcal{R}(m)) c \in H^{1}(Y, \mathcal{S}) \cong \operatorname{Ext}^{1}\left(\varnothing_{Y}, \mathcal{S}\right)$, say $0 \rightarrow \mathcal{S} \rightarrow \mathcal{S}^{\prime} \rightarrow \emptyset_{Y} \rightarrow$ 0 , or $0 \rightarrow V \rightarrow V^{\prime} \rightarrow \mathbb{A}_{Y} \rightarrow 0$ if written for geometric vector bundles with sheaf of sections $\mathcal{S}$. The main equivalence is now that

$$
f_{0} \in\left(f_{1}, \ldots, f_{n}\right)^{\star} \quad \text { if and only if } \mathbb{P}\left(V^{\prime}\right)-\mathbb{P}(V) \text { is not an affine scheme, }
$$

where $\mathbb{P}(V)$ denotes the projective bundle corresponding to the vector bundle $V$ (this is $\mathbb{P}\left(\mathcal{S}^{\vee}\right)$ in the notation of [4] or [8, II.7]). This equivalence rests upon the interpretation of tight closure as solid closure and the geometric interpretation of forcing algebras; see [9] and [1. We will work with solid closure denoted by $I^{\star}$ in the following even if we speak about tight closure. It is the same as tight closure in positive characteristic.

For the affineness of $\mathbb{P}\left(V^{\prime}\right)-\mathbb{P}(V)$ given by a class $c \in H^{1}(Y, \mathcal{S})$ we have proved in 2] several sufficient and necessary slope criteria. Recall that the slope of a locally free sheaf $\mathcal{S}$ is defined by $\mu(\mathcal{S})=\operatorname{deg}(\mathcal{S}) / \operatorname{rank}(\mathcal{S})$. Every locally free sheaf $\mathcal{S}$ has a unique Harder-Narasimhan filtration (see 6], [14], [15]). This is a filtration of locally free subsheaves

$$
0=\mathcal{S}_{0} \subset \mathcal{S}_{1} \subset \cdots \subset \mathcal{S}_{s}=\mathcal{S}
$$

such that $\mathcal{S}_{i} / \mathcal{S}_{i-1}$ is semistable for every $i=1, \ldots, s$. Here $\mathcal{S}_{1}$ is called the maximal destabilizing subsheaf. The slopes of these semistable quotients form a decreasing chain $\mu_{1}>\cdots>\mu_{s}$. We call

$$
\mu_{\min }(\mathcal{S})=\mu_{s}=\mu\left(\mathcal{S} / \mathcal{S}_{s-1}\right)
$$

the minimal slope and

$$
\mu_{\max }(\mathcal{S})=\mu_{1}(\mathcal{S})
$$

the maximal slope. This is the same as

$$
\mu_{\min }(\mathcal{S})=\min \{\mu(\mathcal{Q}): \mathcal{S} \rightarrow \mathcal{Q} \rightarrow 0 \text { locally free quotient sheaf }\}
$$

and

$$
\mu_{\max }(\mathcal{S})=\max \{\mu(\mathcal{T}): \mathcal{T} \subseteq \mathcal{S} \text { locally free subsheaf }\} .
$$

For the dual sheaf we have $\mu_{\max }\left(\mathcal{S}^{\vee}\right)=-\mu_{\min }(\mathcal{S})$. A locally free sheaf $\mathcal{S}$ is called semistable if $\mu_{\min }(\mathcal{S})=\mu_{\max }(\mathcal{S})$.

In positive characteristic we need the following definition. 
Definition 1.1. Let $Y$ denote a smooth projective curve over an algebraically closed field $K$ and let $\mathcal{S}$ denote a locally free sheaf. Then we define

$$
\bar{\mu}_{\max }(\mathcal{S})=\sup \left\{\frac{\mu_{\max }\left(\varphi^{*} \mathcal{S}\right)}{\operatorname{deg}(\varphi)} \mid \varphi: Z \rightarrow Y \text { finite dominant } K \text {-morphism }\right\}
$$

and

$$
\bar{\mu}_{\min }(\mathcal{S})=\inf \left\{\frac{\mu_{\min }\left(\varphi^{*} \mathcal{S}\right)}{\operatorname{deg}(\varphi)} \mid \varphi: Z \rightarrow Y \text { finite dominant } K \text {-morphism }\right\} .
$$

These numbers exist and give nothing new in characteristic zero. In positive characteristic however they may differ from $\mu_{\max }(\mathcal{S})$ and $\mu_{\min }(\mathcal{S})$. We say $\mathcal{S}$ is strongly semistable if $\bar{\mu}_{\max }(\mathcal{S})=\bar{\mu}_{\min }(\mathcal{S})$. This is equivalent to the property that every Frobenius pull-back of $\mathcal{S}$ is semistable; see [18, §5].

With these notions our main slope criteria for affineness are the following.

Theorem 1.2. Let $Y$ denote a smooth projective curve over an algebraically closed field $K$, let $\mathcal{S}$ denote a locally free sheaf on $Y$ and let $c \in H^{1}(Y, \mathcal{S})$ denote a cohomology class given rise to $\mathbb{P}\left(V^{\prime}\right)-\mathbb{P}(V)$. Then the following hold.

(i) Suppose that the characteristic of $K$ is zero. If $\mu_{\max }(\mathcal{S})<0$ and $c \neq 0$, then $\mathbb{P}\left(V^{\prime}\right)-\mathbb{P}(V)$ is affine.

(ii) Suppose that the characteristic of $K$ is zero. Suppose that there exists a sheaf homomorphism $\varphi: \mathcal{S} \rightarrow \mathcal{T}$ such that $\mathcal{T}$ is semistable of negative slope and $0 \neq \varphi(c) \in H^{1}(Y, \mathcal{T})$. Then $\mathbb{P}\left(V^{\prime}\right)-\mathbb{P}(V)$ is affine.

(iii) If $\bar{\mu}_{\min }(\mathcal{S}) \geq 0$, then $\mathbb{P}\left(V^{\prime}\right)-\mathbb{P}(V)$ is not affine.

(iv) If $c=0$, then $\mathbb{P}\left(V^{\prime}\right)-\mathbb{P}(V)$ is not affine.

Proof. See [2, Corollary 3.2, Theorem 3.4, and Theorem 4.4] ((iv) is trivial).

The condition in Theorem 1.2(i) implies that the dual sheaf $\mathcal{S}^{\vee}$ and also the extension $\mathcal{S}^{\prime \vee}$ given by $c \neq 0$ is ample ([3] Theorem 2.2]). This means by definition that the divisor $\mathbb{P}(V) \subset \mathbb{P}\left(V^{\prime}\right)$ is ample and hence its complement is affine. Ampleness and affineness are open properties: if we have a smooth projective relative curve $Y$ over Spec $D$, where $\mathbb{Z} \subseteq D$ is a finitely generated $\mathbb{Z}$-algebra, and if $\mathcal{S}$ is locally free on $Y$, then the affineness of $\mathbb{P}\left(V_{\eta}^{\prime}\right)-\mathbb{P}\left(V_{\eta}\right)$ over the generic point $\eta \in$ Spec $D$ implies the affineness of $\mathbb{P}\left(V^{\prime}\right)-\mathbb{P}(V)$ over an open nonempty subset of Spec $D$. This observation allows us to deduce from results in characteristic zero results for characteristic $p \gg 0$, in particular when the situation is given by a tight closure problem. From these affineness criteria we get the following slope criteria for tight closure.

Theorem 1.3. Let $R$ denote a two-dimensional normal standard-graded domain over an algebraically closed field $K$. Let $\left(f_{1}, \ldots, f_{n}\right)$ denote an $R_{+}$-primary homogeneous ideal given by homogeneous ideal generators of degree $d_{i}=\operatorname{deg}\left(f_{i}\right)$. Let $Y=$ Proj $R$ denote the corresponding smooth projective curve of degree $\delta=$ $\operatorname{deg} \emptyset_{Y}(1)$. Let $\mathcal{R}(m)$ denote the locally free sheaf of relations of total degree $m$. Set $\mu_{\max }\left(f_{1}, \ldots, f_{n}\right):=\mu_{\max }\left(\mathcal{R}(0)^{\vee}\right)$ and define $\bar{\mu}_{\max }\left(f_{1}, \ldots, f_{n}\right), \mu_{\min }\left(f_{1}, \ldots, f_{n}\right)$ and $\bar{\mu}_{\min }\left(f_{1}, \ldots, f_{n}\right)$ in the same way. Let $f_{0}$ denote another homogeneous element. Then the following hold.

(i) If $\operatorname{deg}\left(f_{0}\right) \geq \bar{\mu}_{\max }\left(f_{1}, \ldots, f_{n}\right) / \delta$, then $f_{0} \in\left(f_{1}, \ldots, f_{n}\right)^{\star}$. 
(ii) Suppose that the characteristic of $K$ is zero or $p \gg 0$.

If $\operatorname{deg}\left(f_{0}\right)<\mu_{\min }\left(f_{1}, \ldots, f_{n}\right) / \delta$, then $f_{0} \in\left(f_{1}, \ldots, f_{n}\right)^{\star}$ if and only if $f_{0} \in$ $\left(f_{1}, \ldots, f_{n}\right)$.

(iii) Suppose that the characteristic is zero. If the sheaf of relations $\mathcal{R}(m)$ is semistable, then

$$
\left(f_{1}, \ldots, f_{n}\right)^{\star}=\left(f_{1}, \ldots, f_{n}\right)+R_{\geq \frac{d_{1}+\cdots+d_{n}}{n-1}} .
$$

Proof. These statements follow from Theorem 1.2] see [2, Theorem 6.4, Theorem 7.3 and Theorem 8.1]

Remark 1.4. Note that $\operatorname{det} \mathcal{R}(m)=\emptyset_{Y}\left((n-1) m-d_{1}-\cdots-d_{n}\right)$ and $\operatorname{deg}(\mathcal{R}(m))=$ $\left((n-1) m-d_{1}-\cdots-d_{n}\right) \delta$, where $\delta$ is the degree of the curve $Y$. Therefore

$$
\mu_{\min }\left(f_{1}, \ldots, f_{n}\right) \leq \mu\left(\mathcal{R}(0)^{\vee}\right)=\frac{d_{1}+\cdots+d_{n}}{n-1} \delta \leq \mu_{\max }\left(f_{1}, \ldots, f_{n}\right) .
$$

For the actual computation of tight closure we have to find bounds for the minimal and the maximal slope for the sheaf of relations and criteria for semistability.

\section{AN ALGORITHM FOR LOW RANK}

In this section we describe the first steps of an "algorithm" to decide whether an open subset $\mathbb{P}\left(V^{\prime}\right)-\mathbb{P}(V)$ given by a cohomology class $c \in H^{1}(Y, \mathcal{S})$ is affine or not, where $\mathcal{S}$ is the sheaf of sections in the geometric vector bundle $V$. It always gives a complete answer if the maximal destabilizing subsheaf of $\mathcal{S}$ is semistable, hence in particular if the rank of $\mathcal{S}$ is two. This implies that it is possible to decide whether $f_{0} \in\left(f_{1}, f_{2}, f_{3}\right)^{\star}$ holds or not, at least if we are able to compute the Harder-Narasimhan filtration of the sheaf of relations. We assume that the characteristic of $K$ is zero. The Harder-Narasimhan filtration of $\mathcal{S}$,

$$
0=\mathcal{S}_{0} \subset \mathcal{S}_{1} \subset \cdots \subset \mathcal{S}_{s-1} \subset \mathcal{S}_{s}=\mathcal{S},
$$

splits into short exact sequences

$$
0 \longrightarrow \mathcal{S}_{j-1} \stackrel{i}{\longrightarrow} \mathcal{S}_{j} \longrightarrow \mathcal{S}_{j} / \mathcal{S}_{j-1} \longrightarrow 0,
$$

where the quotients $\mathcal{S}_{j} / \mathcal{S}_{j-1}$ are semistable with slope $\mu_{j}(\mathcal{S})=\mu\left(\mathcal{S}_{j} / \mathcal{S}_{j-1}\right)$. The algorithm uses the fact that for a cohomology class $c_{j} \in H^{1}\left(Y, \mathcal{S}_{j}\right)$ we have either $0 \neq \bar{c}_{j} \in H^{1}\left(Y, \mathcal{S}_{j} / \mathcal{S}_{j-1}\right)$ or $c_{j}=i\left(c_{j-1}\right)$, where $c_{j-1} \in H^{1}\left(Y, \mathcal{S}_{j-1}\right)$. This argumentation scheme requires arbitrary subsheaves, so even if we start with a sheaf of relations $\mathcal{S}=\mathcal{R}\left(f_{1}, \ldots, f_{n}\right)(m)$ and a cohomology class $c \in H^{1}(Y, \mathcal{R}(m))$ given by another homogeneous element, arbitrary sheaves and cohomology classes come naturally into play.

If $s=1$, then $\mathcal{S}$ is semistable and everything is clear by Theorem 1.2(i), (iii). If $s=2$, then we have an exact sequence

$$
0 \longrightarrow \mathcal{S}_{1} \longrightarrow \mathcal{S} \longrightarrow \mathcal{Q} \longrightarrow 0
$$

where $\mathcal{S}_{1}$ and $\mathcal{Q}$ are semistable of different slope. In this case the algorithm gives a complete answer.

We present the algorithm in Figure 1, Note that for $s=2$ we have $\mu_{s-1}(\mathcal{S})=$ $\mu_{1}(\mathcal{S})=\mu_{\max }(\mathcal{S})$. So if this number is $<0$, then we may conclude that $\mathbb{P}\left(V^{\prime}\right)-\mathbb{P}(V)$ is affine by Theorem[1.2(i). 


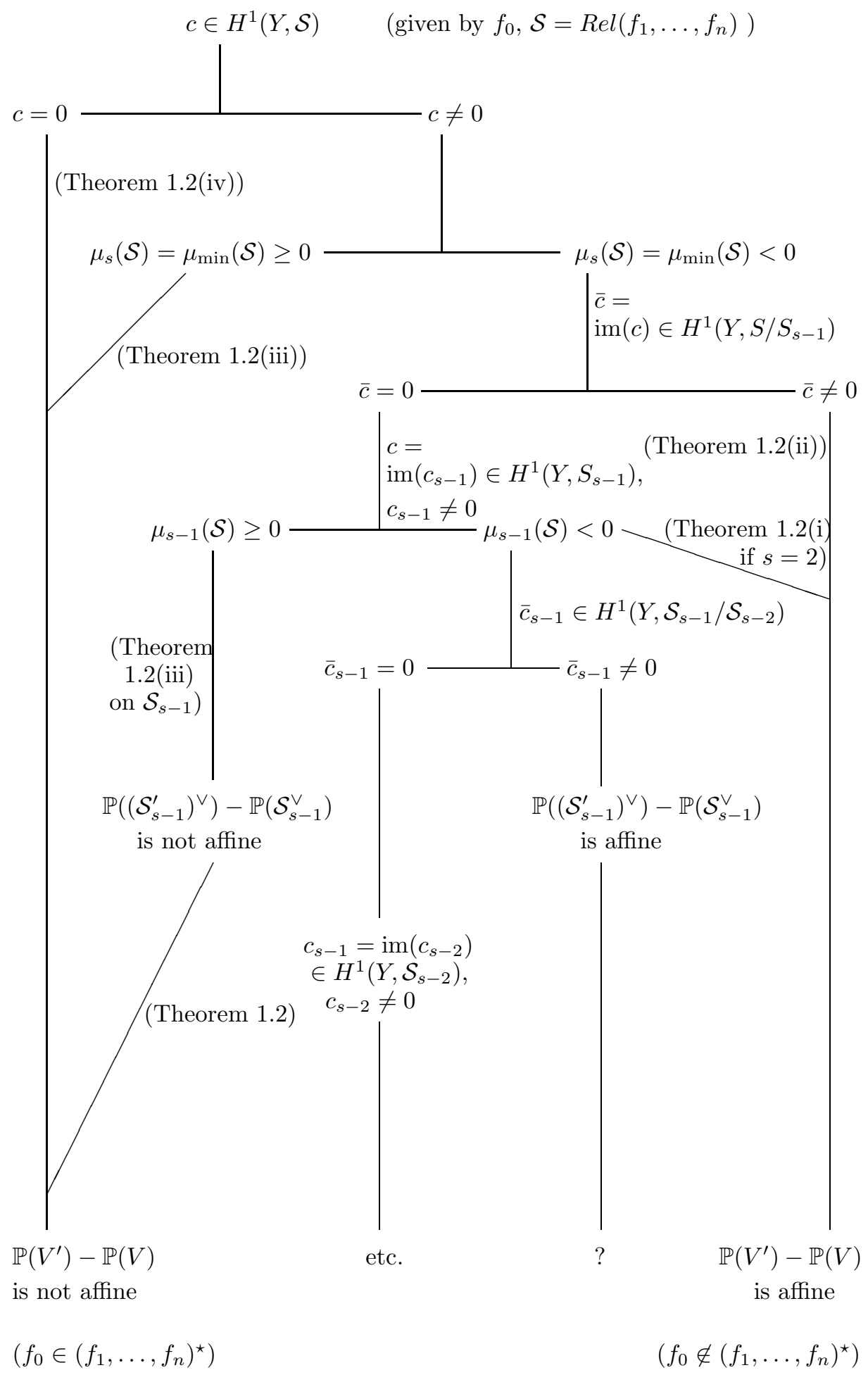

Figure 1. 


\section{The TIGHT ClOSURE OF THREE ELEMENTS}

We fix the following situation.

Situation 3.1. Let $R$ denote a two-dimensional normal standard-graded domain over an algebraically closed field $K$. Let $f_{1}, f_{2}, f_{3} \in R$ denote three homogeneous $R_{+-}$ primary elements of degree $d_{i}=\operatorname{deg}\left(f_{i}\right)$. Let $Y=$ Proj $R$ denote the corresponding smooth projective curve of degree $\delta=\operatorname{deg} \varnothing_{Y}(1)$ and of genus $g$ and let $\mathcal{R}(m)$ denote the sheaf of relations of total degree $m$ for $f_{1}, f_{2}, f_{3}$.

The sheaf of relations $\mathcal{R}(m)$ on $Y$ has rank two; hence we may decide in characteristic zero due to Section 2 whether $\mathbb{P}\left(V^{\prime}\right)-\mathbb{P}(V)$ (given by the cohomology class $\left.\delta\left(f_{0}\right)=c \in H^{1}(Y, \mathcal{R}(m))\right)$ is affine or not. Therefore we may compute $\left(f_{1}, f_{2}, f_{3}\right)^{\star}$, at least if we can compute the Harder-Narasimhan filtration of $\mathcal{R}$.

In positive characteristic we have to refine this algorithm, since the HarderNarasimhan filtration is in general not stable under the Frobenius pull-back. For a locally free sheaf $\mathcal{S}$ we set

$$
\lambda_{1}(\mathcal{S})=\max \{\operatorname{deg}(\mathcal{L}): \mathcal{L} \text { is an invertible subsheaf of } \mathcal{S}\}
$$

and

$$
\rho_{1}(\mathcal{S})=\min \{\operatorname{deg}(\mathcal{L}): \mathcal{L} \text { is an invertible quotient sheaf of } \mathcal{S}\} .
$$

For a locally free sheaf $\mathcal{S}$ of rank two we have $\mu_{\max }(\mathcal{S})=\max \left\{\lambda_{1}(\mathcal{S}), \operatorname{deg}(\mathcal{S}) / 2\right\}$ and $\mu_{\min }(\mathcal{S})=\min \left\{\rho_{1}(\mathcal{S}), \operatorname{deg}(\mathcal{S}) / 2\right\}$, and $\rho_{1}(\mathcal{S})=\operatorname{deg}(\mathcal{S})-\lambda_{1}(\mathcal{S})$. The sheaf $\mathcal{S}$ is semistable if and only if $\lambda_{1}(\mathcal{S}) \leq \operatorname{deg}(\mathcal{S}) / 2$.

If we find a subsheaf $\mathcal{L} \subseteq \mathcal{S}$ such that $\operatorname{deg}(\mathcal{L}) \geq \operatorname{deg}(\mathcal{S}) / 2$ and such that the quotient is itself locally free (i.e., $\mathcal{L}$ is a subbundle), then $\operatorname{deg}(\mathcal{L})=\lambda_{1}(\mathcal{S})=\mu_{\max }(\mathcal{S})$.

Lemma 3.2. Let $\mathcal{S}$ denote a locally free sheaf of rank two on a smooth projective curve $Y$. Suppose that we have a short exact sequence $0 \rightarrow \mathcal{L} \rightarrow \mathcal{S} \rightarrow \mathcal{M} \rightarrow$ 0 , where $\mathcal{L}$ and $\mathcal{M}$ are invertible sheaves. Then $\lambda_{1}(\mathcal{S}) \leq \max (\operatorname{deg}(\mathcal{L}), \operatorname{deg}(\mathcal{M}))$. If furthermore $\operatorname{deg}(\mathcal{L}) \geq \mu(\mathcal{S})=\operatorname{deg}(\mathcal{S}) / 2$, then $\lambda_{1}(\mathcal{S})=\operatorname{deg}(\mathcal{L})$ and $\rho_{1}(\mathcal{S})=$ $\operatorname{deg}(\mathcal{M})$.

If $\operatorname{deg}(\mathcal{L})=\mu(\mathcal{S})$, then $\mathcal{S}$ is strongly semistable. If $\operatorname{deg}(\mathcal{L})>\mu(\mathcal{S})$, then $\mathcal{S}$ is not stable and $\mathcal{L}$ is the maximal destabilizing subsheaf.

Proof. Let $\mathcal{N}$ denote an invertible sheaf and let $\varphi: \mathcal{N} \rightarrow \mathcal{S}$ be a sheaf morphism. If $\operatorname{deg}(\mathcal{N})>\operatorname{deg}(\mathcal{M})$, then the composed morphism $\mathcal{N} \rightarrow \mathcal{M}$ is zero and $\varphi$ factors through $\mathcal{L}$. But then $\operatorname{deg}(\mathcal{N}) \leq \operatorname{deg}(\mathcal{L})$ or $\varphi$ is zero. So suppose that $\operatorname{deg}(\mathcal{L}) \geq \mu(\mathcal{S})$. Then $\operatorname{deg}(\mathcal{M})=\operatorname{deg}(\mathcal{S})-\operatorname{deg}(\mathcal{L}) \leq \operatorname{deg}(\mathcal{S})-\operatorname{deg}(\mathcal{S}) / 2=\operatorname{deg}(\mathcal{S}) / 2$, and hence $\operatorname{deg}(\mathcal{L}) \geq \mu(\mathcal{S}) \geq \operatorname{deg}(\mathcal{M})$. Thus $\mathcal{L}$ is a subbundle of maximal degree and $\mathcal{M}$ is a quotient invertible sheaf of minimal degree. The other statements follow.

We now have the following two alternatives. The locally free sheaf $\mathcal{S}$ of rank two on $Y$ is strongly semistable. Then $\bar{\mu}_{\max }(\mathcal{S})=\mu(\mathcal{S})=\bar{\mu}_{\min }(\mathcal{S})$ and the tight closure is easy to compute by the numerical criterion Theorem 1.3. Otherwise $\mathcal{S}$ is not strongly semistable. Then there exists a finite morphism $\varphi: Y^{\prime} \rightarrow Y$ such that there exists a short exact sequence on $Y^{\prime}, 0 \rightarrow \mathcal{L} \rightarrow \mathcal{S}^{\prime} \rightarrow \mathcal{M} \rightarrow 0$, where $\operatorname{deg}(\mathcal{L}) \geq \mu\left(\mathcal{S}^{\prime}\right), \mathcal{S}^{\prime}=\varphi^{*}(\mathcal{S})$. In this case the pull-back of this sequence for another morphism $\psi: Y^{\prime \prime} \rightarrow Y^{\prime}$ also fulfills the condition in Lemma [3.2; hence $\mu_{\max }\left(\mathcal{S}^{\prime \prime}\right)=\operatorname{deg}\left(\psi^{*}(\mathcal{L})\right)=\operatorname{deg}(\mathcal{L}) \operatorname{deg}(\psi)$ and thus $\bar{\mu}_{\max }(\mathcal{S})=\operatorname{deg}(\mathcal{L}) / \operatorname{deg}(\varphi)$. 
If we have a short exact sequence for the sheaf of relations for three elements $f_{1}, f_{2}, f_{3}$, then we can often compute $\left(f_{1}, f_{2}, f_{3}\right)^{\star}$ and $\left(f_{1}, f_{2}, f_{3}\right)^{+}$(the plus closure) according to the following proposition.

Proposition 3.3. Assume the notation of Situation 3.1. Let $0 \rightarrow \mathcal{L} \rightarrow \mathcal{R}(m) \rightarrow$ $\mathcal{M} \rightarrow 0$ denote a short exact sequence, where $\mathcal{L}$ and $\mathcal{M}$ are invertible. Let $f_{0}$ denote another homogeneous element of degree $m$ and let $c \in H^{1}(Y, \mathcal{R}(m))$ denote its forcing class. Let $\bar{c}$ denote the image of $c$ in $H^{1}(Y, \mathcal{M})$. Then the following hold (suppose in the first two statements that the characteristic of $K$ is zero or $p \gg 0$ ).

(i) If $\operatorname{deg}(\mathcal{L})<0$ and $\operatorname{deg}(\mathcal{M})<0$ and $c \neq 0$, then $f_{0} \notin\left(f_{1}, f_{2}, f_{3}\right)^{\star}$.

(ii) If $\operatorname{deg}(\mathcal{M})<0$ and $\bar{c} \neq 0$, then $f_{0} \notin\left(f_{1}, f_{2}, f_{3}\right)^{\star}$.

(iii) If $\bar{c}=0$ and $\operatorname{deg}(\mathcal{L}) \geq 0$, then $f_{0} \in\left(f_{1}, f_{2}, f_{3}\right)^{\star}$.

(iv) If $\operatorname{deg}(\mathcal{L}) \geq \operatorname{deg}(\mathcal{M}) \geq 0$, then $f_{0} \in\left(f_{1}, f_{2}, f_{3}\right)^{\star}$.

(v) Suppose that the characteristic of $K$ is positive. Suppose that $\bar{c}=0$ or that $\mathcal{M}$ is trivial or that $\mathcal{M}$ has positive degree. Moreover suppose that $\mathcal{L}$ is trivial or has positive degree. Then $f_{0} \in\left(f_{1}, f_{2}, f_{3}\right)^{+}$.

Proof. We use the equivalence that $f_{0} \notin\left(f_{1}, f_{2}, f_{3}\right)^{\star}$ if and only if $\mathbb{P}\left(V^{\prime}\right)-\mathbb{P}(V)$ is affine (1.1), where $V$ is the vector bundle corresponding to the sheaf of relations and $V^{\prime}$ is the extension of $V$ given by the forcing class.

(i) The maximal slope of $\mathcal{R}(m)$ is $<0$; hence the result follows from Theorem $1.2(\mathrm{i})$.

(ii) This follows from Theorem 1.2 (ii).

(iii) If $\bar{c}=0$, then there exists a cohomology class $e \in H^{1}(Y, \mathcal{L})$ mapping to $c$. Let $\mathcal{L}^{\prime}$ denote the extension of $\mathcal{L}$ defined by this cohomology class $e$. If $f_{0} \notin\left(f_{1}, f_{2}, f_{3}\right)^{\star}$, then $\mathbb{P}\left(V^{\prime}\right)-\mathbb{P}(V)$ would be affine and then $\mathbb{P}\left(\left(\mathcal{L}^{\prime}\right)^{\vee}\right)-\mathbb{P}\left(\mathcal{L}^{\vee}\right) \subset \mathbb{P}\left(V^{\prime}\right)-\mathbb{P}(V)$ would be affine as a closed subscheme, but this is not true since $\operatorname{deg}(\mathcal{L}) \geq 0$ and Theorem 1.2 (iii).

(iv) The condition implies in connection with Lemma 3.2 that $\bar{\mu}_{\min }(\mathcal{R}(m)) \geq 0$; hence the result follows again from Theorem 1.2 (iii).

(v) After applying a finite mapping $\varphi: Y^{\prime} \rightarrow Y$, we may assume that $\bar{c}=0$. For $\operatorname{deg}(\mathcal{M})>0$ this can be done by a Frobenius power and for $\mathcal{M}=\varnothing_{Y}$ this is due to 1, Proposition 8.1]. Therefore we may assume that $\varphi^{*}(c)$ stems from a cohomology class $e \in H^{1}\left(Y^{\prime}, \varphi^{*}(\mathcal{L})\right)$. Due to the assumptions on $\mathcal{L}$ we can do the same with $e$; hence there exists altogether a finite mapping $Y^{\prime \prime} \rightarrow Y$ such that the pull-back of $c$ is zero. Therefore $f_{0} \in\left(f_{1}, f_{2}, f_{3}\right)^{+}$.

We may apply Proposition 3.3 to the short exact sequence given by the HarderNarasimhan filtration to compute the tight closure of $\left(f_{1}, f_{2}, f_{3}\right)$ if $\mathcal{R}$ is not strongly stable.

Corollary 3.4. Assume the notation of Situation 3.1. Suppose that the sheaf of relations $\mathcal{R}$ is not strongly stable, and let $\varphi: Y^{\prime} \rightarrow Y$ denote a finite dominant morphism of smooth projective curves such that there exists a short exact sequence

$$
0 \longrightarrow \mathcal{L}(m) \longrightarrow \varphi^{*}(\mathcal{R}(m)) \longrightarrow \mathcal{M}(m) \longrightarrow 0
$$

on $Y^{\prime}$, where $\operatorname{deg}(\mathcal{L}(m)) \geq \mu\left(\varphi^{*}(\mathcal{R}(m))\right) \geq \operatorname{deg}(\mathcal{M}(m))$. Let $f_{0}$ denote another homogeneous element of degree $m$, let $c$ denote its forcing class in $H^{1}\left(Y^{\prime}, \varphi^{*}(\mathcal{R}(m))\right)$ and let $\bar{c}$ denote its image in $H^{1}(Y, \mathcal{M}(m))$. Then we may decide whether $f_{0} \in$ $\left(f_{1}, f_{2}, f_{3}\right)^{\star}$ in the following way (assume in the first and second statements that the characteristic is zero or $p \gg 0)$. 
(i) If $\operatorname{deg}(\mathcal{L}(m))<0$ and $c \neq 0$, then $f_{0} \notin\left(f_{1}, f_{2}, f_{3}\right)^{\star}$.

(ii) If $\operatorname{deg}(\mathcal{L}(m)) \geq 0, \operatorname{deg}(\mathcal{M}(m))<0$ and $\bar{c} \neq 0$, then $f_{0} \notin\left(f_{1}, f_{2}, f_{3}\right)^{\star}$.

(iii) If $\operatorname{deg}(\mathcal{L}(m)) \geq 0, \operatorname{deg}(\mathcal{M}(m))<0$ and $\bar{c}=0$, then $f_{0} \in\left(f_{1}, f_{2}, f_{3}\right)^{\star}$.

(iv) If $\operatorname{deg}(\mathcal{M}(m)) \geq 0$, then $f_{0} \in\left(f_{1}, f_{2}, f_{3}\right)^{\star}$.

Proof. This follows from Proposition 3.3 .

Remark 3.5. Assume the conditions of Corollary 3.4 and suppose that the characteristic is positive. Then we need in (iii) and (iv) stronger conditions to conclude that $f_{0} \in\left(f_{1}, f_{2}, f_{3}\right)^{+}$. For (iii) we need that $\mathcal{L}(m)$ is trivial or that $\operatorname{deg}(\mathcal{L}(m))$ is positive. For (iv) we need that $\mathcal{M}$ is of positive degree. This follows from Proposition $3.3 \mathrm{v}$ ).

If the sheaf of relations is decomposable, that is, it is the sum of two invertible sheaves, then the decomposition gives at once a short exact sequence and Proposition 3.3 and Corollary 3.4 are particularly easy to apply. On the other hand we cannot expect any bound for $\mu_{\max }$ in the decomposable case. For the indecomposable case we have the following result.

Theorem 3.6. Assume the notation of Situation 3.1 and suppose that the characteristic of $K$ is zero. Suppose that the sheaf of relations $\mathcal{R}(m)$ is indecomposable on $Y$. Then

$$
\mu_{\max }\left(f_{1}, f_{2}, f_{3}\right) \leq \delta \frac{d_{1}+d_{2}+d_{3}}{2}+g-1
$$

and

$$
\mu_{\min }\left(f_{1}, f_{2}, f_{3}\right) \geq \delta \frac{d_{1}+d_{2}+d_{3}}{2}-g+1 .
$$

Proof. See [2, Theorem 9.1].

\section{The DEgREe of RELATions}

The notions of semistability and of minimal and maximal degree of a locally free sheaf $\mathcal{S}$ on a smooth projective curve $Y$ refer to all locally free subsheaves of $\mathcal{S}$ (or quotient sheaves). However for a relation sheaf $\mathcal{R}(m)$ defined by homogeneous primary elements $f_{1}, \ldots, f_{n}$ in a two-dimensional normal standard-graded $K$-domain $R$ we have the fixed polarization $\emptyset_{Y}(1)$ on $Y=\operatorname{Proj} R$. It is then often easier to control the behavior of $\mathcal{R}(m)=\mathcal{R}(0) \otimes \emptyset_{Y}(m)$ instead of $\mathcal{R}(0) \otimes \mathcal{L}$ for all invertible sheaves $\mathcal{L}$. The (non)existence of relations $\neq 0$ for $f_{1}, \ldots, f_{n}$ of certain degree has many consequences on the structure of $\mathcal{R}(m)$ and hence on the corresponding tight closure problem.

Remark 4.1. The global relations of $\mathcal{R}(m)$ obey the theorem of Riemann-Roch, that is,

$$
h^{0}(Y, \mathcal{R}(m))-h^{1}(Y, \mathcal{R}(m))=\left((n-1) m-d_{1}-\cdots-d_{n}\right) \delta+(1-g)(n-1),
$$

where $\delta=\operatorname{deg} \varnothing_{Y}(1)$ is the degree of $Y$ and $g$ is its genus. In particular, for $m>\frac{d_{1}+\cdots+d_{n}}{n-1}+\frac{g-1}{\delta}$ there exist global relations $\neq 0$ in $\mathcal{R}(m)$.

Lemma 4.2. Suppose that the locally free sheaf $\mathcal{S}$ on the smooth projective curve $Y$ of genus $g$ over an algebraically closed field $K$ does not have sections $\neq 0$. Then $\Gamma(Y, \mathcal{S} \otimes \mathcal{L})=0$ for every invertible sheaf $\mathcal{L}$ of degree $\leq-$ g. In particular $\lambda_{1}(\mathcal{S}) \leq$ $g-1$. 
Proof. Suppose the contrary. Then we have a nontrivial morphism $\mathcal{M} \rightarrow \mathcal{S}$ such that $\operatorname{deg} \mathcal{M} \geq g$. But due to the theorem of Riemann-Roch we have $h^{0}(\mathcal{M}) \geq$ $\operatorname{deg}(\mathcal{M})+1-g$; hence the invertible sheaf $\mathcal{M}$ must have nontrivial sections, which gives a contradiction.

Proposition 4.3. Let $f_{1}, \ldots, f_{n}$ denote homogeneous primary elements in a normal two-dimensional standard-graded $K$-domain $R$ of degree $d_{i}$, where $K$ is an algebraically closed field. Suppose that $Y=\operatorname{Proj} R$ has genus $g=g(Y)$ and degree $\delta$.

(i) Suppose that there exists a relation $\neq 0$ for the elements $f_{1}, \ldots, f_{n}$ of total degree $k<\left(d_{1}+\cdots+d_{n}\right) /(n-1)$. Then the sheaf of relations is not semistable.

(ii) Suppose that there does not exist a relation $\neq 0$ of total degree $k$. Then

$$
\lambda_{1}(\mathcal{R}(k)) \leq g-1 .
$$

(iii) Let $n=3$. Suppose that there exists a relation $\neq 0$ of total degree $k<$ $\frac{d_{1}+d_{2}+d_{3}}{2}-\frac{g-1}{\delta}$. Then the sheaf of relations is decomposable, i.e., the sum of two invertible sheaves.

(iv) Let $n=3$ and suppose that there does not exist a relation $\neq 0$ of total degree $k \geq \frac{d_{1}+d_{2}+d_{3}}{2}+\frac{g-1}{\delta}$. Then $\mathcal{R}$ is semistable.

Proof. (i) The relation $\neq 0$ induces a nontrivial morphism $\varnothing_{Y} \rightarrow \mathcal{R}(k)$, but the degree $\operatorname{deg}(\mathcal{R}(k))=\left((n-1) k-d_{1}-\cdots-d_{n}\right) \delta<0$ is negative; hence $\mathcal{R}(k)$ is not semistable.

(ii) The assumption means that $\mathcal{S}=\mathcal{R}(k)$ has no global sections $\neq 0$; hence Lemma 4.2 yields that $\lambda_{1}(\mathcal{R}(k)) \leq g-1$.

(iii) Since $\mathcal{R}(k)$ has a nontrivial section, the sheaf $\mathcal{R}(k)$ contains the structure sheaf as a subsheaf and therefore $\mu_{\max }(\mathcal{R}(k)) \geq 0$. On the other hand we have $\mu(\mathcal{R}(k))+g-1=\left(k-\frac{d_{1}+d_{2}+d_{3}}{2}\right) \delta+g-1<0$, that is, $\mu(\mathcal{R}(k))<1-g$. Therefore we have a short exact sequence $0 \rightarrow \mathcal{L} \rightarrow \mathcal{R}(k) \rightarrow \mathcal{M} \rightarrow 0$ where $\operatorname{deg}(\mathcal{L}) \geq 0$ and $\operatorname{deg}(\mathcal{M})<2(1-g)$. This extension corresponds to a class $H^{1}\left(Y, \mathcal{L} \otimes \mathcal{M}^{-1}\right) \cong$ $H^{0}\left(Y, \mathcal{L}^{-1} \otimes \mathcal{M} \otimes \omega_{Y}\right)=0$; hence it is trivial.

(iv) The numerical condition means that $g-1 \leq\left(k-\frac{1}{2}\left(d_{1}+d_{2}+d_{3}\right)\right) \delta=$ $\operatorname{deg}(\mathcal{R}(k)) / 2$; hence from (ii) we get that $\lambda_{1}(\mathcal{R}(k)) \leq \operatorname{deg}(\mathcal{R}(k)) / 2$ and the sheaf of relations is semistable.

We may derive from Proposition 4.3(ii) the following inclusion bound for tight closure.

Corollary 4.4. Assume the notation of Situation 3.1 and that the characteristic of $K$ is zero. Suppose that there does not exist a relation $\neq 0$ of total degree $k \leq \frac{d_{1}+d_{2}+d_{3}}{2}+\frac{g-1}{\delta}$. Then $R_{m} \subseteq\left(f_{1}, f_{2}, f_{3}\right)^{\star}$ holds for $m \geq d_{1}+d_{2}+d_{3}-k+\frac{g-1}{\delta}$.

Proof. If $\mathcal{R}$ is semistable, then the result follows from Theorem 1.3(iii). If $\mathcal{R}$ is not semistable, then $\mu_{\max }(\mathcal{R}(k))=\lambda_{1}(\mathcal{R}(k)) \leq g-1$ by Proposition 4.3 (ii) and we get

$$
\begin{aligned}
\mu_{\max }\left(f_{1}, f_{2}, f_{3}\right) & =\left(d_{1}+d_{2}+d_{3}\right) \delta-\mu_{\min }\left(f_{1}, f_{2}, f_{3}\right) \\
& =\left(d_{1}+d_{2}+d_{3}\right) \delta+\mu_{\max }(\mathcal{R}(0)) \\
& =\left(d_{1}+d_{2}+d_{3}\right) \delta+\mu_{\max }(\mathcal{R}(k))-k \delta \\
& \leq\left(d_{1}+d_{2}+d_{3}-k\right) \delta+g-1 .
\end{aligned}
$$


Hence under the numerical condition we have $\operatorname{deg}\left(f_{0}\right)=m \geq \mu_{\max }\left(f_{1}, f_{2}, f_{3}\right) / \delta$ and the result follows from Theorem[1.3(i).

Corollary 4.5. Let $R=K[x, y, z] /(F)$ denote a normal two-dimensional standardgraded $K$-domain over an algebraically closed field $K$, where $F$ is a polynomial of degree $\delta$. Let $f_{1}, f_{2}, f_{3} \in R$ be $R_{+}$-primary homogeneous elements of degree $d_{1}, d_{2}, d_{3}$. Suppose that there does not exist a relation $\neq 0$ for $f_{1}, f_{2}, f_{3}$ of total degree $k$ with $k \geq \frac{d_{1}+d_{2}+d_{3}}{2}+\frac{\delta-3}{2}$. Then $\mathcal{R}$ is semistable.

Proof. This follows directly from Proposition 4.3 (iv).

Remark 4.6. Corollary 4.5 is only applicable for $k=\frac{d_{1}+d_{2}+d_{3}}{2}+\frac{\delta-3}{2}$, since for greater $k$ there exist global relations due to Remark 4.1. The same is true for Proposition 4.3(iv).

Example 4.7. We consider the elements $x^{d}, y^{d}, z^{d}$ on a smooth projective curve given by an equation $F=0$, where $F$ is a homogeneous polynomial of degree $\delta$. There exist relations like $\left(y^{d},-x^{d}, 0\right)$ of total degree $2 d$. Suppose that there do not exist relations of smaller degree. Then the numerical condition in Corollary 4.5 for semistability is that $2 d-1 \geq 3 d / 2+(\delta-3) / 2$ or equivalently that $\delta \leq d+1$. If we want to apply Corollary 4.5 we have to make sure that the defining polynomial $F$ of degree $\delta$ does not yield relations of degree $<2 d$.

Look at $d=2$ and $\delta=3$. If the monomial $x y z$ does occur in $F$, then there do not exist relations of degree 3 and the relation sheaf is semistable. However this yields nothing interesting for tight closure, since then $x y z \in\left(x^{2}, y^{2}, z^{2}\right)$ holds anyway.

Now look at $d=4$ and $\delta=5$. Under suitable conditions for the coefficients of $F$ there does not exist a relation of degree 7 for $x^{4}, y^{4}, z^{4}$. Write $F=a x^{3} y^{2}+$ $b x^{3} y z+c x^{3} z^{2}+d x^{2} y^{3}+\cdots$. A relation of degree 7 is the same as a multiple $F Q$ $(\operatorname{deg}(Q)=2)$ which belongs to $\left(x^{4}, y^{4}, z^{4}\right)$. The six monomials of degree 2 yield six linear combinations in the six monomials of degree 7 outside $\left(x^{4}, y^{4}, z^{4}\right)$, namely $x^{3} y^{3} z, x^{3} y^{2} z^{2}, x^{3} y z^{3}, x^{2} y^{3} z^{2}, x^{2} y^{2} z^{3}$ and $x y^{3} z^{3}$. We may choose the coefficients of $F$ in such a way that these linear combinations are linearly independent. Then there does not exist a relation of degree 7 . So in this case the sheaf of relations is semistable. It follows for $\operatorname{char}(K)=0$ that $R_{6} \subseteq\left(x^{4}, y^{4}, z^{4}\right)^{\star}$. Note that it is not true that $R_{6} \subseteq\left(x^{4}, y^{4}, z^{4}\right)$, since there exist 10 monomials of degree 6 outside $\left(x^{4}, y^{4}, z^{4}\right)$ in $K[x, y, z]$; hence the dimension of $R_{6} /\left(x^{4}, y^{4}, z^{4}\right)$ is at least $10-3$.

Remark 4.8. If $\mathcal{S}$ is a locally free sheaf of rank two, then we have the natural mapping $\mathcal{S} \oplus \mathcal{S} \rightarrow \mathcal{S} \wedge \mathcal{S} \cong \operatorname{det} \mathcal{S}$. This mapping induces an isomorphism $\mathcal{S} \cong$ $\mathcal{S}^{\vee} \otimes \operatorname{det} \mathcal{S}$. If $\mathcal{S}=\mathcal{R}(m)$ is the sheaf of relations for three homogeneous elements $f_{1}, f_{2}, f_{3}$ of degree $d_{i}$, then

$\mathcal{R}(m)^{\vee} \cong \mathcal{R}(m) \otimes \operatorname{det} \mathcal{R}(m)^{\vee}=\mathcal{R}(m) \otimes \emptyset_{Y}\left(d_{1}+d_{2}+d_{3}-2 m\right)=\mathcal{R}\left(d_{1}+d_{2}+d_{3}-m\right)$.

The natural mapping $\mathcal{R}(m) \oplus \mathcal{R}(m) \rightarrow \operatorname{det}(\mathcal{R}(m)) \cong \emptyset_{Y}\left(2 m-d_{1}-d_{2}-d_{3}\right)$ is in terms of the injection $\mathcal{R}(m) \subset \varnothing_{Y}\left(m-d_{1}\right) \oplus \varnothing_{Y}\left(m-d_{2}\right) \oplus \varnothing_{Y}\left(m-d_{3}\right)$ given by

$$
\left(g_{1}, g_{2}, g_{3}\right),\left(h_{1}, h_{2}, h_{3}\right) \longmapsto \frac{g_{2} h_{3}-g_{3} h_{2}}{f_{1}}=\frac{-g_{1} h_{3}+g_{3} h_{1}}{f_{2}}=\frac{g_{1} h_{2}-g_{2} h_{1}}{f_{3}} .
$$

A global primary relation $\left(g_{1}, g_{2}, g_{3}\right) \in \mathcal{R}(m)$ yields the quotient mapping $\mathcal{R}(m) \rightarrow$ $\varnothing_{Y}\left(2 m-d_{1}-d_{2}-d_{3}\right)$ which is given by $\left(h_{1}, h_{2}, h_{3}\right) \mapsto \frac{-h_{2} g_{3}+h_{3} g_{2}}{f_{1}}$. 
If moreover $f_{1}$ and $f_{2}$ are parameters, then the forcing class $c=\delta(f)$ of an element $f$ of degree $m$ is represented by the Čech-cocycle

$$
\left(\frac{f}{f_{1}},-\frac{f}{f_{2}}, 0\right) \in H^{1}(Y, \mathcal{R}(m)) .
$$

The quotient mapping sends this class to $-\frac{f g_{3}}{f_{1} f_{2}} \in H^{1}\left(Y, \varnothing_{Y}\left(2 m-d_{1}-d_{2}-d_{3}\right)\right)$.

Example 4.9. The existence of relations depends heavily on the characteristic. Consider $x^{100}, y^{100}, z^{100}$ on $x^{4}+y^{4}+z^{4}=0$. For $\operatorname{char}(K)=5$ we find

$$
\left(x^{4}+y^{4}+z^{4}\right)^{25}=x^{100}+y^{100}+z^{100} ;
$$

hence there exists a relation of total degree 100 and in fact $z^{100} \in\left(x^{100}, y^{100}\right)$ and therefore $\left(x^{100}, y^{100}, z^{100}\right)^{\star}=\left(x^{100}, y^{100}\right)^{\star}$.

For $\operatorname{char}(K)=37$ we have $\left(x^{4}+y^{4}+z^{4}\right)^{37}=x^{48} x^{100}+y^{48} y^{100}+z^{48} z^{100}$, which is a relation of total degree 148 . This relation yields a short exact sequence

$$
0 \longrightarrow \varnothing_{Y}(4) \longrightarrow \mathcal{R}(152) \longrightarrow \varnothing_{Y} \longrightarrow 0,
$$

where the last mapping is given by $\left(g_{1}, g_{2}, g_{3}\right) \mapsto \frac{x^{48} g_{2}-y^{48} g_{1}}{z^{100}}$ due to Remark 4.8 Due to Proposition 4.3(iii) the sheaf of relations is decomposable, and we show that this sequence splits.

We may write $z^{100}=\left(-x^{4}-y^{4}\right)^{25}=x^{48} g_{2}-y^{48} g_{1}$ where $g_{1}$ and $g_{2}$ have degree 52 . We can build a relation $\left(g_{1}, g_{2}, g_{3}\right)$ of degree 152 by $g_{3}=\frac{-\left(g_{1} x^{100}+g_{2} y^{100}\right)}{z^{100}}$, for then

$$
\begin{aligned}
g_{3}=\frac{-\left(g_{1} x^{100}+g_{2} y^{100}\right)}{z^{100}} & =\frac{-\left(g_{1} x^{148}+g_{2} y^{100} x^{48}\right)}{x^{48} z^{100}} \\
& =\frac{g_{1}\left(y^{148}+z^{148}\right)-g_{2} y^{100} x^{48}}{x^{48} z^{100}} \\
& =\frac{g_{1} z^{48}}{x^{48}}+\frac{g_{1} y^{148}-g_{2} y^{100} x^{48}}{x^{48} z^{100}} \\
& =\frac{g_{1} z^{48}}{x^{48}}-\frac{y^{100}}{x^{48}} .
\end{aligned}
$$

Therefore $g_{3}$ may be written with denominators $z$ and $x$; hence it is a global section of $\emptyset_{Y}(52)$. Hence $\left(g_{1}, g_{2}, g_{3}\right)$ is a global relation of total degree 152 which maps to 1 , so the short exact sequence splits.

The nonexistence of global relations implies the ampleness of the dual sheaf of the sheaf of relations. This observation then yields exclusion criteria for tight closure.

Proposition 4.10. Let $K$ denote an algebraically closed field of characteristic zero and let $R$ denote a two-dimensional normal standard-graded domain. Suppose that $f_{1}, \ldots, f_{n} \in R$ are $R_{+}$-primary homogeneous elements. Suppose that there does not exist a global relation $\neq 0$ of total degree $k$ for the elements $f_{1}, \ldots, f_{n}$. Set $\mathcal{F}(-m):=\mathcal{R}(m)^{\vee}$. Then $\mathcal{F}(-m)$ is ample for $m<k-\frac{2 n-3}{(n-1) \delta} g+\frac{1}{\delta}$. In particular, $\mathcal{F}(-m)$ is ample for $m \leq k-2 g / \delta$.

Proof. We know by Proposition 4.3(ii) that $\lambda_{1}(\mathcal{R}(k)) \leq g-1$ and therefore dually that $\rho_{1}(\mathcal{F}(-k)) \geq-g+1$. Hence

$\rho_{1}(\mathcal{F}(-m))=\rho_{1}\left(\mathcal{F}(-k) \otimes \emptyset_{Y}(k-m)\right)=\rho_{1}(\mathcal{F}(-k))+(k-m) \delta \geq-g+1+(k-m) \delta$.

The numerical condition is equivalent to $-g+1+(k-m) \delta>\frac{n-2}{n-1} g$. Hence $\rho_{1}(\mathcal{F}(-m))>\frac{n-2}{n-1} g$ and the result follows from [2, Corollary 2.2]. 
Corollary 4.11. Let $R=K[x, y, z] /(F)$ be a normal standard-graded $K$-domain over an algebraically closed field $K$ of characteristic zero, where $F$ is an irreducible polynomial of degree $\delta$. Let $f_{1}, \ldots, f_{n}$ denote primary homogeneous elements of degree $d_{i}$. Suppose that there does not exist a global relation $\neq 0$ for $f_{1}, \ldots, f_{n}$ of total degree $k$. Then $\mathcal{F}(-m)$ is ample for $m \leq k-\delta+2$. An element $f_{0} \in R$ of degree $m \leq k-\delta+2$ belongs to $\left(f_{1}, \ldots, f_{n}\right)^{\star}$ if and only if it belongs to $\left(f_{1}, \ldots, f_{n}\right)$.

Proof. We have $m \leq k-\delta+2 \leq k-(\delta-2)(\delta-1) / \delta=k-2 g / \delta$; hence the ampleness of $\mathcal{F}(-m)$ for $m \leq k-\delta+2$ follows from Proposition 4.10, Now if $f$ is a homogeneous element of degree $m \leq k-\delta+2$ such that $f \notin\left(f_{1}, \ldots, f_{n}\right)$, then $f$ defines a nontrivial extension $0 \rightarrow \emptyset \rightarrow \mathcal{F}^{\prime} \rightarrow \mathcal{F}(-m) \rightarrow 0$ and therefore $\mathcal{F}^{\prime}$ is also ample (characteristic zero). Therefore $\mathbb{P}\left(\mathcal{F}^{\prime}\right)-\mathbb{P}(\mathcal{F}(-m))$ is affine and $f \notin\left(f_{1}, \ldots, F_{n}\right)^{\star}$ by $(1.1)$.

Example 4.12. We want to apply Proposition 4.10 and Corollary 4.11 to Example 4.7 for $d=4, \delta=5$ under the condition that there does not exist a global relation of degree 7 .

Then Corollary 4.11 shows $(k=7, g=6, n=3)$ that $\mathcal{F}(-m)$ is ample only for $m \leq 7-5+2=4$. The second bound in Proposition 4.10 gives this for $m \leq 7-\frac{2 \cdot 6}{5}=4.6$. The first bound in Proposition 4.10 however yields ampleness for $m<7-\frac{3}{2 \cdot 5} 6+\frac{1}{5}=7-\frac{16}{10}=5.4$. Therefore $\left(x^{4}, y^{4}, z^{4}\right)^{\star} \cap R_{m}=\left(x^{4}, y^{4}, z^{4}\right) \cap R_{m}$ for $m \leq 5$.

\section{The existence of PRIMARY RElations}

We suppose further that $\mathcal{R}$ is the sheaf of relations on a smooth projective curve $Y=\operatorname{Proj} R$ for homogeneous primary elements $f_{1}, \ldots, f_{n} \in R$, where $R$ is a twodimensional normal standard-graded $K$-domain over an algebraically closed field $K$. We say that a relation $r \in \Gamma(Y, \mathcal{R}(m))$ is a primary relation if it has no zero on $Y$ or, equivalently, if $r: \varnothing_{Y} \rightarrow \mathcal{R}(m)$ defines a subbundle. For a primary relation we get a short exact sequence $0 \rightarrow \emptyset_{Y} \rightarrow \mathcal{R}(m) \rightarrow \mathcal{Q} \rightarrow 0$, where $\mathcal{Q}$ is also locally free.

Corollary 5.1. Assume the notation of Situation 3.1. Suppose that there exists a primary relation of total degree $k$. Then this relation gives rise to a short exact sequence

$$
0 \rightarrow \varnothing_{Y} \rightarrow \mathcal{R}(k) \rightarrow \varnothing_{Y}\left(2 k-d_{1}-d_{2}-d_{3}\right) \rightarrow 0 .
$$

If $k \geq \frac{d_{1}+d_{2}+d_{3}}{2}$, then $R_{m} \subseteq\left(f_{1}, f_{2}, f_{3}\right)^{\star}$ holds for $m \geq k$. The same holds in positive characteristic for the plus closure.

If $k \leq\left(d_{1}+d_{2}+d_{3}\right) / 2$, then $\lambda_{1}(\mathcal{R}(k))=0$ and $\rho_{1}(\mathcal{R}(k))=\left(2 k-d_{1}-d_{2}-d_{3}\right) \delta \leq 0$.

If moreover $k=\left(d_{1}+d_{2}+d_{3}\right) / 2$, then the sheaf of relations is strongly semistable.

Proof. The primary relation yields an invertible quotient sheaf which is isomorphic to $\operatorname{det} \mathcal{R}(k)$. For $k \geq \frac{d_{1}+d_{2}+d_{3}}{2}$ this quotient sheaf has degree $\geq 0$ and we are in the situation of Proposition 3.3 (iv) and (v).

Now suppose $k \leq\left(d_{1}+d_{2}+d_{3}\right) / 2$. We have $\operatorname{deg}\left(\varnothing_{Y}\right)=0 \geq k-\left(d_{1}+d_{2}+d_{3}\right) / 2=$ $\mu(\mathcal{R}(k))$; hence we are in the situation of Lemma 3.2. If $k=\left(d_{1}+d_{2}+d_{3}\right) / 2$, then $\mathcal{R}(k)$ is the extension of the structure sheaf by itself; hence its degree is 0 and this follows again from Lemma 3.2 
Example 5.2. Consider a Fermat polynomial $x^{k}+y^{k}+z^{k} \in K[x, y, z]$ and let $R=K[x, y, z] /\left(x^{k}+y^{k}+z^{k}\right)$. Let $f=x^{d_{1}}, g=y^{d_{2}}, h=z^{d_{3}}$ such that $d_{i} \leq k$ and $d_{1}+d_{2}+d_{3} \leq 2 k$. Then $\left(x^{k-d_{1}}, y^{k-d_{2}}, z^{k-d_{3}}\right)$ is a primary relation of total degree $k$. Therefore by Corollary 5.1 we get $R_{\geq k} \subseteq\left(x^{d_{1}}, y^{d_{2}}, z^{d_{3}}\right)^{\star}$ and we also get $R_{\geq k} \subseteq\left(x^{d_{1}}, y^{d_{2}}, z^{d_{3}}\right)^{+\mathrm{gr}}$ in positive characteristic.

Corollary 5.3. Assume the notation of Situation 3.1. Suppose that there exists a primary relation of total degree $k \leq\left(d_{1}+d_{2}+d_{3}\right) / 2$. Suppose that the characteristic of the algebraically closed field $K$ is zero or $p \gg 0$. Let $f_{0} \in R$ be a homogeneous element of degree $\operatorname{deg}\left(f_{0}\right)=m$. Then the following hold.

If $m<k$, then $f_{0} \in\left(f_{1}, f_{2}, f_{3}\right)^{\star}$ if and only if $f_{0} \in\left(f_{1}, f_{2}, f_{3}\right)$.

If $m \geq d_{1}+d_{2}+d_{3}-k$, then $f_{0} \in\left(f_{1}, f_{2}, f_{3}\right)^{\star}$.

Proof. From Corollary 5.1 we get the short exact sequence $0 \rightarrow \varnothing_{Y}(m-k) \rightarrow$ $\mathcal{R}(m) \rightarrow \varnothing_{Y}\left(m+k-d_{1}-d_{2}-d_{3}\right) \rightarrow 0$, where $\operatorname{deg}\left(\varnothing_{Y}(m-k)\right) \geq \mu(\mathcal{R}(m)) \geq$ $\operatorname{deg}\left(\varnothing_{Y}\left(m+k-d_{1}-d_{2}-d_{3}\right)\right)$. Thus we are in the situation of Corollary 3.4(i) and (iv).

We may also deduce a result about the plus closure.

Corollary 5.4. Assume the notation of Situation 3.1 and suppose that $K$ has positive characteristic. Suppose that there exists a primary relation of total degree $k \leq\left(d_{1}+d_{2}+d_{3}\right) / 2$. Then $\left(f_{1}, f_{2}, f_{3}\right)^{\star}=\left(f_{1}, f_{2}, f_{3}\right)^{+\mathrm{gr}}$.

Proof. Let $c \in H^{1}(Y, \mathcal{R}(m))$ denote the cohomology class of a homogeneous element $f_{0} \in R$ of degree $m$. We look at the sequence from Corollary 5.1, $0 \rightarrow \varnothing_{Y}(m-k) \rightarrow$ $\mathcal{R}(m) \rightarrow \varnothing_{Y}\left(m+k-d_{1}-d_{2}-d_{3}\right) \rightarrow 0$ and run through the cases according to Corollary 3.4 If $m \geq d_{1}+d_{2}+d_{3}-k$, then $f_{0} \in\left(f_{1}, f_{2}, f_{3}\right)^{+}$follows from Proposition 3.3 v). So suppose that $m<d_{1}+d_{2}+d_{3}-k$. If the image of $c$ in $H^{1}\left(Y, \varnothing_{Y}\left(m+k-d_{1}-d_{2}-d_{3}\right)\right) \neq 0$, then $f_{0} \notin\left(f_{1}, f_{2}, f_{3}\right)^{\star}$ by Proposition 3.3(i). So we may assume that $c$ stems from $e \in H^{1}\left(Y, \varnothing_{Y}(m-k)\right)$. If $m \geq k$ or $c=0$, then the pull-back of $e$ under a finite mapping is zero; hence $f_{0} \in\left(f_{1}, f_{2}, f_{3}\right)^{+}$. If $m<k$ and $c \neq 0$, then $f_{0} \notin\left(f_{1}, f_{2}, f_{3}\right)^{\star}$ by Proposition 3.3(ii).

Example 5.5. Consider the ideal $\left(x^{10}, y^{10}, z^{10}\right)$ on the curve given by the equation $x^{4}+y^{4}=z^{4}$. Due to Remark 4.1 there exists a relation $\neq 0$ of degree 16 . We have

$$
z^{16}=\left(x^{4}+y^{4}\right)^{4}=x^{16}+4 x^{12} y^{4}+6 x^{8} y^{8}+4 x^{4} y^{12}+y^{16}
$$

and

$$
x^{4} z^{12}=x^{4}\left(x^{4}+y^{4}\right)^{3}=x^{16}+3 x^{12} y^{4}+3 x^{8} y^{8}+x^{4} y^{12} .
$$

Therefore we may write

$z^{10}\left(z^{6}-2 z^{2} x^{4}\right)=-x^{16}-2 x^{12} y^{4}+2 x^{4} y^{12}+y^{16}=x^{10}\left(-x^{6}-2 x^{2} y^{4}\right)+y^{10}\left(2 x^{4} y^{2}+y^{6}\right) ;$

hence we have the relation $\left(z^{6}-2 z^{2} x^{4}, x^{6}+2 x^{2} y^{4},-2 x^{4} y^{2}-y^{6}\right)$ of total degree 16. This relation is primary: if $x=0$ or $y=0$, then $x=y=z=0$, so suppose $x, y \neq 0$. Then $x^{4}+2 y^{4}=0$ and $2 x^{4}+y^{4}=0$ which gives $3 x^{4}=0$; hence the relation has no common zero (in characteristic $\neq 3$ ) and is therefore primary. We have therefore the short exact sequence

$$
0 \longrightarrow \varnothing_{Y} \longrightarrow \mathcal{R}(16) \longrightarrow \varnothing_{Y}(2) \longrightarrow 0
$$


and it follows from Corollary [5.1 that $R_{\geq 16} \subset\left(x^{10}, y^{10}, z^{10}\right)^{\star}$. We tensor this short exact sequence with $\varnothing(k-16)$ and dualize it to get

$$
0 \longrightarrow \varnothing_{Y}(14-k) \longrightarrow \mathcal{R}^{\vee}(-k) \longrightarrow \varnothing_{Y}(16-k) \longrightarrow 0 \text {. }
$$

This shows that $\mathcal{R}^{\vee}(-k)$ is ample for $k \leq 13$ as an extension of two ample invertible sheaves; therefore $\left(x^{10}, y^{10}, z^{10}\right)^{\star} \cap R_{\leq 13}=\left(x^{10}, y^{10}, z^{10}\right) \cap R_{\leq 13}$ holds in characteristic 0 and $p \gg 0$. If there does not exist a relation of degree 15, then this also holds for $k=14$ due to Proposition 4.10.

Example 5.6. Let $K$ denote an algebraically closed field and consider

$$
R=K[x, y, z] /\left(x^{\delta}+a y^{\delta}+b z^{\delta}+c x z^{\delta-1}+d y z^{\delta-1}\right)
$$

where $a, b, c, d \neq 0$ are chosen such that $Y=\operatorname{Proj} R$ is smooth. Consider the relation sheaf for the elements $x^{\delta}, y^{\delta}, z^{\delta}$. Then we have a relation of total degree $\delta+1$, given by $(z, a z, b z+c x+d y)$. Since $\delta+1<\frac{3 \delta}{2}-\frac{\delta-3}{2}=\frac{3 \delta}{2}-\frac{g-1}{\delta}$, it follows from Proposition 4.3 (iii) that $\mathcal{R}$ is decomposable.

The relation $(z, a z, b z+c x+d y)$ is primary if and only if $c x+d y$ and $x^{\delta}+a y^{\delta}$ have no common homogeneous zero. This is true if and only if $\left(-\frac{d}{c}\right)^{\delta} \neq-a$. If this is true, then we have the splitting $\mathcal{R}(\delta+1)=\emptyset_{Y} \oplus \varnothing_{Y}(-\delta+2)$, where the second summand corresponds to a relation of total degree $2 \delta-1$. We can find such a relation in the following way: There exists a polynomial $P(x, y)$ in $x$ and $y$ of degree $\delta-1$ such that $(c x+d y) P(x, y)=r x^{\delta}+s y^{\delta}$. Then $\left(P+r z^{\delta-1}, a P+s z^{\delta-1}, b P\right)$ is a relation of total degree $2 \delta-1$, since $P x^{\delta}+a P y^{\delta}+r x^{\delta} z^{\delta-1}+s y^{\delta} z^{\delta-1}+b P z^{\delta}=$ $P x^{\delta}+a P y^{\delta}+P b z^{\delta}+P c x z^{\delta-1}+P d y z^{\delta-1}=0$.

Corollary 5.7. Let $f_{1}, f_{2}, f_{3} \in K[x, y, z]$ be homogeneous polynomials of degree $d_{1}, d_{2}, d_{3}$ such that $d_{1}+d_{2}+d_{3}=2 k$ is even and $k \geq d_{i}$ for $i=1,2,3$. Let $g_{1}, g_{2}, g_{3} \in K[x, y, z]$ be homogeneous of degree $k-d_{i}$. Suppose that $V\left(f_{1}, f_{2}, f_{3}\right)=$ $V\left(g_{1}, g_{2}, g_{3}\right)=V(x, y, z)$. Set $F=f_{1} g_{1}+f_{2} g_{2}+f_{3} g_{3}$ and suppose that $R=$ $K[x, y, z] /(F)$ is a normal domain. Then the sheaf of relations $\mathcal{R}(m)$ for $f_{1}, f_{2}, f_{3}$ on $Y=\operatorname{Proj} R$ is an extension of the structure sheaf by itself and is strongly semistable. In particular

$$
\left(f_{1}, f_{2}, f_{3}\right)^{\star}=R_{\geq k}+\left(f_{1}, f_{2}, f_{3}\right) .
$$

If furthermore the characteristic of $K$ is positive, then $\left(f_{1}, f_{2}, f_{3}\right)^{\star}=\left(f_{1}, f_{2}, f_{3}\right)^{+\mathrm{gr}}$.

Proof. The relation $\left(g_{1}, g_{2}, g_{3}\right)$ is primary of total degree $k$; thus this follows from Corollary [5.1.

\section{The tight Closure of $\left(x^{a}, y^{a}, z^{a}\right)$ IN $K[x, y, z] /(F)$}

In this section we study the tight closure of $\left(x^{a}, y^{a}, z^{a}\right)$ in $R=K[x, y, z] /(F)$, where $F$ is an irreducible homogeneous polynomial of degree $\delta$ such that $R$ is normal. The expected generic answer is by Theorem 1.3(iii) that $\left(x^{a}, y^{a}, z^{a}\right)^{\star}=$ $\left(x^{a}, y^{a}, z^{a}\right)+R_{\geq \frac{3}{2} a}$. We have however to check that the sheaf of relations is semistable to obtain this result in characteristic zero, and in positive characteristic we have to do even more. Sullivant has made some computations implemented in Macaulay2 for the monomial ideals $\left(x^{a}, y^{a}, z^{a}\right)^{\star}$ for $a=2,3,4$ for small prime numbers $p$ and small degree $\delta$ for the Fermat equations $x^{\delta}+y^{\delta}+z^{\delta}=0$ (see [23]). These computations have led him to conjectures about the behavior of the tight closure; we will prove some of his conjectures. 
The homogeneous ideal generators $x^{a}, y^{a}, z^{a}$ yield the sheaf of relations $\mathcal{R}(m)=$ $\operatorname{Rel}\left(x^{a}, y^{a}, z^{a}\right)(m)$ on the smooth projective curve $Y=\operatorname{Proj} R \subset \mathbb{P}^{2}$. This sheaf is the restriction of the sheaf of relations $\mathcal{R}_{\mathbb{P}^{2}}(m)=\operatorname{Rel}_{\mathbb{P}^{2}}\left(x^{a}, y^{a}, z^{a}\right)(m)$ on the projective plane. On $\mathbb{P}^{2}$ we have the presenting sequence

$$
0 \longrightarrow \mathcal{R}_{\mathbb{P}^{2}}(m) \longrightarrow \bigoplus_{3} \emptyset_{\mathbb{P}^{2}}(m-a) \stackrel{x^{a}, y^{a}, z^{a}}{\longrightarrow} \emptyset_{\mathbb{P}^{2}}(m) \longrightarrow 0
$$

and the exact sequence (from the Koszul complex)

$$
0 \longrightarrow \emptyset_{\mathbb{P}^{2}}(m-3 a) \longrightarrow \bigoplus_{3} \emptyset_{\mathbb{P}^{2}}(m-2 a) \longrightarrow \mathcal{R}_{\mathbb{P}^{2}}(m) \longrightarrow 0,
$$

where the surjection is given by the standard relations

$$
\left(-y^{a}, x^{a}, 0\right), \quad\left(z^{a}, 0,-x^{a}\right), \quad\left(0,-z^{a}, y^{a}\right)
$$

and the injection by $1 \mapsto\left(z^{a}, y^{a}, x^{a}\right)$. Since the sheaves in these short exact sequences are locally free, their restrictions to a curve are also exact (they are subbundles).

Lemma 6.1. Let $F \in K[x, y, z]$ denote an irreducible homogeneous polynomial of degree $\delta$ and suppose that it defines a smooth projective curve $Y$. Let $\mathcal{R}(m)$ denote the sheaf of relations on $Y$ for the elements $x^{a}, y^{a}, z^{a}, a \geq 1$. Then every relation $\in \Gamma(Y, \mathcal{R}(k))$ for $k<\delta$ is a linear combination of the three standard relations $\left(-y^{a}, x^{a}, 0\right),\left(z^{a}, 0,-x^{a}\right)$ and $\left(0,-z^{a}, y^{a}\right)$. In particular $\Gamma(Y, \mathcal{R}(k))=0$ for $k<$ $2 a, \delta$.

Proof. On $\mathbb{P}^{2}$ we have the exact sequence

$$
0 \longrightarrow \emptyset_{\mathbb{P}^{2}}(-a) \longrightarrow \bigoplus_{3} \emptyset_{\mathbb{P}^{2}} \longrightarrow \mathcal{R}_{\mathbb{P}^{2}}(2 a) \longrightarrow 0
$$

where the surjection is given by the standard relations. This surjection is also globally a surjection. We tensor the exact sequence for $Y \subset \mathbb{P}^{2}$, that is,

$$
0 \longrightarrow \emptyset_{\mathbb{P}^{2}}(-\delta) \stackrel{F}{\longrightarrow} \emptyset_{\mathbb{P}^{2}} \longrightarrow \emptyset_{Y} \longrightarrow 0
$$

with $\mathcal{R}_{\mathbb{P}^{2}}(k)$, and by applying $\Gamma\left(\mathbb{P}^{2},-\right)$, we get

$$
\Gamma\left(\mathbb{P}^{2}, \mathcal{R}_{\mathbb{P}^{2}}(k)\right) \longrightarrow \Gamma\left(\mathbb{P}^{2}, \mathcal{R}_{Y}(k)\right) \longrightarrow H^{1}\left(\mathbb{P}^{2}, \mathcal{R}_{\mathbb{P}^{2}}(k-\delta)\right) .
$$

We want to show that the term on the right is zero. By the presenting sequence for the relations we get

$$
0 \longrightarrow \mathcal{R}_{\mathbb{P}^{2}}(k-\delta) \longrightarrow \bigoplus_{3} \varnothing_{\mathbb{P}^{2}}(k-\delta-a) \longrightarrow \varnothing_{\mathbb{P}^{2}}(k-\delta) \longrightarrow 0
$$

and hence

$$
\Gamma\left(\mathbb{P}^{2}, \emptyset_{\mathbb{P}^{2}}(k-\delta)\right) \longrightarrow H^{1}\left(\mathbb{P}^{2}, \mathcal{R}_{\mathbb{P}^{2}}(k-\delta)\right) \longrightarrow \bigoplus_{3} H^{1}\left(\mathbb{P}^{2}, \emptyset_{\mathbb{P}^{2}}(k-\delta-a)\right)=0
$$

shows that $\Gamma\left(\mathbb{P}^{2}, \mathcal{R}_{\mathbb{P}^{2}}(k)\right) \longrightarrow \Gamma\left(\mathbb{P}^{2}, \mathcal{R}_{Y}(k)\right)$ is surjective for $k<\delta$. Then the statements follow since they are true on $\mathbb{P}^{2}$.

Proposition 6.2. Let $F \in K[x, y, z]$ denote a polynomial of degree $\delta \geq 3 a-1$ and suppose that it defines a smooth projective curve $Y$. Then the sheaf of relations $\mathcal{R}(m)$ for the elements $x^{a}, y^{a}, z^{a}$ is semistable. 
Proof. Suppose that there exists an invertible subsheaf $\mathcal{L} \subset \mathcal{R}(\delta-1)$ of degree $>\mu(\mathcal{R}(\delta-1))=\frac{2(\delta-1)-3 a}{2} \delta=\left(\delta-1-\frac{3}{2} a\right) \delta$. Then

$$
\begin{aligned}
\operatorname{deg}(\mathcal{L})+1-g & >\left(\delta-1-\frac{3}{2} a\right) \delta+1-\frac{(\delta-1)(\delta-2)}{2} \\
& =\delta\left(\delta-1-\frac{3}{2} a-\frac{\delta-3}{2}\right)=\delta\left(\frac{\delta}{2}-\frac{3}{2} a+\frac{1}{2}\right) .
\end{aligned}
$$

This expression is $\geq 0$ for $\delta \geq 3 a-1$; hence due to the theorem of Riemann-Roch the invertible sheaf $\mathcal{L}$ has global sections $\neq 0$. Therefore we look at the global sections of $\mathcal{R}(\delta-1)$ and study their zeros in order to get a bound for the maximal degree of a subbundle.

We use the inclusion $\mathcal{R}(\delta-1) \subset \varnothing_{Y}(\delta-a-1) \oplus \varnothing_{Y}(\delta-a-1) \oplus \varnothing_{Y}(\delta-a-1)$ and think of a global relation $S \in \Gamma(Y, \mathcal{R}(\delta-1))$ as given by three polynomials $\left(S_{1}, S_{2}, S_{3}\right)$ of degree $\delta-a-1$. By Lemma 6.1 we know that the global relations of total degree $\delta-1$ are of the form

$$
\left(S_{1}, S_{2}, S_{3}\right)=A\left(-y^{a}, x^{a}, 0\right)+B\left(-z^{a}, 0, x^{a}\right)+C\left(0, z^{a},-y^{a}\right),
$$

where $A, B, C$ are homogeneous polynomials in $K[x, y, z]$ of degree $\delta-2 a-1$. A relation $\varnothing_{Y} \rightarrow \mathcal{R}(\delta-1)$ has a zero if and only if the three components $S_{1}=$ $A y^{a}+B z^{a}, S_{2}=A x^{a}+C z^{a}$ and $S_{3}=B x^{a}-C y^{a}$ have a common zero. We have to show that the number of zeros (with multiplicities) of such a relation is bounded by the slope of $\mathcal{R}(\delta-1)$, that is, by $\left(\delta-1-\frac{3}{2} a\right) \delta$. We write

$$
S_{1}=A y^{a}+B z^{a}=Q_{1} P \quad \text { and } \quad S_{2}=A x^{a}+C z^{a}=Q_{2} P,
$$

where $Q_{1}, Q_{2}$ have no common divisor, $\operatorname{deg}\left(Q_{1}\right)=\operatorname{deg}\left(Q_{2}\right)=t$. We assume first that $a \leq t \leq \delta-a-1$. The number of zeros of the relation on the curve given by $F=0$ is then bounded by ( $F$ and $P$ have no common divisor, since $F$ is irreducible of degree $\delta>\operatorname{deg}(P))$

$$
\operatorname{deg}(P) \delta+\operatorname{deg}\left(Q_{1}\right) \operatorname{deg}\left(Q_{2}\right)=(\delta-a-1-t) \delta+t^{2} .
$$

We claim that $t^{2} \leq \delta\left(t-\frac{1}{2} a\right)$. We can check this at the boundaries $t=a$ and $t=\delta-a-1$. For $t=a$ this is the inequality $a^{2} \leq \delta a / 2$, which is true since $\delta \geq 3 a-1 \geq 2 a$ for $a \geq 1$. For $t=\delta-a-1$ we have to show that $(\delta-a-1)(\delta-a-1) \leq$ $\delta\left(\delta-\frac{3}{2} a-1\right)$, which yields the condition $a^{2}+2 a+1 \leq \delta(1+a / 2)$. But this is true since $\delta \geq 3 a-1$ and $\delta \geq 1$.

The claim implies that $-\delta(a+t)+t^{2} \leq-\frac{3}{2} a \delta$. Therefore we have

$$
(\delta-a-1-t) \delta+t^{2}=(\delta-1) \delta-\delta(a+t)+t^{2} \leq(\delta-1) \delta-\frac{3}{2} a \delta .
$$

Assume now that $t<a$. We may assume that the powers $z^{b}, b \geq a$, do not occur in the polynomial $A$, since $z^{a}\left(-y^{a}, x^{a}, 0\right)=y^{a}\left(-z^{a}, 0, x^{a}\right)+x^{a}\left(0, z^{a},-y^{a}\right)$. From $Q_{2} S_{1}=Q_{1} S_{2}$ we obtain the equation

$$
A\left(Q_{1} x^{a}-Q_{2} y^{a}\right)=z^{a}\left(Q_{2} B-Q_{1} C\right) .
$$

If $z$ divides $Q_{1} x^{a}-Q_{2} y^{a}$, then it would also (since $\operatorname{deg}\left(Q_{1}\right)=t<a$ ) divide $Q_{1}$ and $Q_{2}$, but they are coprime. Hence $z^{a}$ divides $A$, but then $A=0$. Therefore $Q_{2} B=Q_{1} C$ and hence $B=Q_{1} D$ and $C=Q_{2} D$, where $D$ is a polynomial of degree $\delta-2 a-1-t$. A zero of the relation is given by $D Q_{1} z^{a}=0, D Q_{2} z^{a}=0$ and $D Q_{1} x^{a}-D Q_{2} y^{a}=0$, hence by $D=0$ or by $Q_{1} z^{a}=Q_{2} z^{a}=Q_{1} x^{a}-Q_{2} y^{a}=0$. The polynomials $Q_{1} z^{a}$ and $Q_{1} x^{a}-Q_{2} y^{a}$ do not have a common divisor, since $Q_{1}$ and 
$Q_{2}$ are coprime and since $z$ does not divide $Q_{1} x^{a}-Q_{2} y^{a}$. Therefore the number of zeros is bounded by $(\delta-2 a-1-t) \delta+(t+a)^{2}$. Again we have to check that this is $\leq\left(\delta-1-\frac{3}{2} a\right) \delta$ and this is equivalent to $(t+a)^{2} \leq \delta\left(\frac{1}{2} a+t\right)$. But this is true for $t=a-1$ and $t=0$.

Corollary 6.3. Let $K$ denote an algebraically closed field of characteristic zero. Let $F \in K[x, y, z]$ denote an irreducible homogeneous polynomial of degree $\delta$ and suppose that $R=K[x, y, z] /(F)$ is normal. Then for $\delta \geq 3 a-1$ we have

$$
\left(x^{a}, y^{a}, z^{a}\right)^{\star}=\left(x^{a}, y^{a}, z^{a}\right)+R_{\geq \frac{3}{2} a} .
$$

Proof. This follows from Proposition 6.2 and Theorem 1.3(iii).

We also obtain from Proposition 6.2 results in positive characteristic. We deduce first the following ampleness result.

Corollary 6.4. Let $F \in K[x, y, z]$ denote an irreducible homogeneous polynomial of degree $\delta$ such that $R=K[x, y, z] /(F)$ is normal. Let $\mathcal{R}(m)$ denote the sheaf of relations for the elements $x^{a}, y^{a}, z^{a}$ of total degree $m$ on $Y=\operatorname{Proj} R$. Suppose that $\delta \geq 3 a-1$ and that the characteristic of $K$ is zero or $p \geq \delta-3$. Then $\mathcal{R}(m)$ is an ample sheaf for $m>\frac{3}{2} a$.

Proof. We will use the ampleness criterion of Hartshorne-Mumford for locally free sheaves of rank two on a smooth projective curve $Y$ (see [7, Proposition 7.5 and Corollary 7.7]). It states that $\mathcal{S}$ is ample provided that

(i) $\operatorname{deg}(\mathcal{S})>\frac{2}{p}(g-1)$, where $g$ is the genus of $Y$,

(ii) every invertible quotient sheaf $\mathcal{S} \rightarrow \mathcal{L} \rightarrow 0$ has positive degree

(in characteristic zero the first condition is just that $\operatorname{deg}(\mathcal{S})>0$ ). We have $\operatorname{deg}(\mathcal{R}(m))=(2 m-3 a) \delta>\delta$ and $g-1=\delta(\delta-3) / 2$; therefore the first condition is fulfilled for $p \geq \delta-3$. The second condition follows from Proposition 6.2 by semistability we have $\operatorname{deg}(\mathcal{L}) \geq \mu(\mathcal{R}(m))>0$.

Corollary 6.5. Let $K$ denote an algebraically closed field of positive characteristic $p$ and let $F \in K[x, y, z]$ denote a homogeneous polynomial of degree $\delta$ such that $R=K[x, y, z] /(F)$ is a normal domain. Suppose that $\delta \geq 3 a-1$. Then the following hold for the Frobenius closure and the tight closure of $\left(x^{a}, y^{a}, z^{a}\right)$.

(i) $R_{m} \subset\left(x^{a}, y^{a}, z^{a}\right)^{F}$ for $m>\frac{3}{2} a$ and $p \geq \delta-3$.

(ii) $R_{m} \subset\left(x^{a}, y^{a}, z^{a}\right)^{\star}$ for $m>\frac{3}{2}$ a and $p \geq \delta-3$.

(iii) $\left(x^{a}, y^{a}, z^{a}\right)^{\star} \cap R_{m}=\left(x^{a}, y^{a}, z^{a}\right) \cap R_{m}$ for $m<\frac{3}{2} a$ and $p \gg 0$.

Proof. (i) We know by Corollary 6.4 that the sheaf of relations $\mathcal{R}(m)$ is ample for $m>\frac{3}{2} a$ and $p \geq \delta-3$. Let $c \in H^{1}(Y, \mathcal{R}(m)$ ) denote a cohomology class (given by an element $\left.f_{0} \in R_{m}\right)$. An ample sheaf $\mathcal{S}$ on a smooth projective curve over an algebraically closed field of positive characteristic is also cohomologically $p$-ample. This means that for every coherent sheaf $\mathcal{F}$ we have $H^{i}\left(Y, \mathcal{S}^{(q)} \otimes \mathcal{F}\right)=0$ for $i \geq 1$ and $q \gg 0$, where $\mathcal{S}^{(q)}$ denotes the Frobenius pull-back of $\mathcal{S}$ (see [7], [17]). In particular the mapping $H^{1}(Y, \mathcal{R}(m)) \rightarrow H^{1}\left(Y, q^{*} \mathcal{R}(m)\right)=H^{1}\left(Y, \mathcal{R}\left(x^{a q}, y^{a q}, z^{a q}\right)(q m)\right)$ is 0 for $q \gg 0$. Hence $c^{q}=0$ and therefore $f^{q} \in\left(x^{a q}, y^{a q}, z^{a q}\right)$. This means that $f$ belongs to the Frobenius closure of $\left(x^{a}, y^{a}, z^{a}\right)$.

(ii) This follows from (i).

(iii) We have $\mathcal{R}(m)^{\vee}=\mathcal{R}(m) \otimes \varnothing_{Y}(3 a-2 m)=\mathcal{R}(3 a-m)$, so for $m<\frac{3}{2} a$ the dual sheaf $\mathcal{F}(-m)=\mathcal{R}(m)^{\vee}$ is ample. A cohomology class $c \in H^{1}(Y, \mathcal{R}(m))$ defines 
an extension $0 \rightarrow \varnothing_{Y} \rightarrow \mathcal{F}^{\prime} \rightarrow \mathcal{F}(-m) \rightarrow 0$. In characteristic zero and for $c \neq 0$ this extension $\mathcal{F}^{\prime}$ is also an ample sheaf. This is then also true for $p \gg 0$; hence the complement $\mathbb{P}\left(\mathcal{F}^{\prime}\right)-\mathbb{P}(\mathcal{F}(-m))$ is an affine scheme for $p \gg 0$. This means that $f \notin\left(x^{a}, y^{a}, z^{a}\right)^{\star}$ for $p \gg 0$ with the exception that $c=\delta(f)=0$, which means that $f \in\left(x^{a}, y^{a}, z^{a}\right)$.

Remark 6.6. Note the difference between $a$ even and $a$ odd. For $a$ odd the critical value $\frac{3}{2} a$ is not the degree of a polynomial and so Corollary 6.5 gives a complete answer for the tight closure of $\left(x^{a}, y^{a}, z^{a}\right)$. This also explains that Sullivant's algorithm works much better for degree 3 than for degree 2 or 4 .

Remark 6.7. The result in Corollary 6.5)(i) is under the much stronger condition $m>2 a$ and is easy to prove using the Koszul resolution. This yields the surjection

$$
\bigoplus_{3} H^{1}\left(Y, \varnothing_{Y}((m-2 a) q)\right) \rightarrow H^{1}\left(Y, \mathcal{R}\left(x^{a q}, y^{a q}, z^{a q}\right)(m q)\right) \rightarrow 0
$$

and $h^{1}\left(\varnothing_{Y}((m-2 a) q)\right)=h^{0}\left(\varnothing_{Y}((2 a-m) q) \otimes \varnothing_{Y}(\delta-3)\right)=0$ for $q \gg 0$.

Remark 6.8. The results above imply in particular Sullivant's Conjectures 4.2 and 4.5 about the behavior of $\left(x^{3}, y^{3}, z^{3}\right)^{\star}(a=3)$ in the Fermat rings for $\delta \geq 8$ and $p \gg 0$. For degree $\delta \geq 4$, see Section 7 .

We close this section with an easy observation concerning the strong semistability of the sheaf of relations.

Proposition 6.9. Let $\mathcal{R}(m)$ denote the sheaf of relations for the elements $x^{a}, y^{a}$, $z^{a}$ on the Fermat curve given by $x^{\delta}+y^{\delta}+z^{\delta}=0$ over an algebraically closed field of positive characteristic $p$. Suppose that $\frac{3 a}{2} q>\delta q^{\prime} \geq a q$ for prime powers $q$ and $q^{\prime}$ of $p$. Then $\mathcal{R}$ is not strongly semistable.

Proof. The pull-back of $\mathcal{R}\left(x^{a}, y^{a}, z^{a}\right)(0)$ under the $k$-th Frobenius morphism, $q=$ $p^{k}$, is $\mathcal{R}\left(x^{a q}, y^{a q}, z^{a q}\right)(0)$. Since $\delta q^{\prime} \geq a q$, we can use the $q^{\prime}$-power of the curve equation, that is, $x^{\delta q^{\prime}}+y^{\delta q^{\prime}}+z^{\delta q^{\prime}}=0$ directly as the relation $\left(x^{\delta q^{\prime}-a q}, y^{\delta q^{\prime}-a q}, z^{\delta q^{\prime}-a q}\right)$ for $x^{a q}, y^{a q}, z^{a q}$ of total degree $\delta q^{\prime}$. This gives a nontrivial morphism $\emptyset_{Y} \rightarrow$ $\mathcal{R}\left(x^{a q}, y^{a q}, z^{a q}\right)\left(\delta q^{\prime}\right)$. The slope on the right is $\delta q^{\prime}-\frac{3}{2} a q<0$, so this sheaf of relations is not semistable.

Remark 6.10. The condition in Proposition 6.9 means either $\frac{3 a}{2} p^{k}>\delta \geq a p^{k}$ or $\frac{3 a}{2}>\delta p^{k} \geq a$. For $\delta p^{k} \geq \frac{3}{2} a$ we get from a similar argument that $R_{\geq \delta p^{k}} \subset$ $\left(x^{a}, y^{a}, z^{a}\right)^{\star}$ (and even in the plus closure).

\section{The tight Closure of $\left(x^{2}, y^{2}, z^{2}\right)$ And $\left(x^{3}, y^{3}, z^{3}\right)$}

We continue with the computation of $\left(x^{a}, y^{a}, z^{a}\right)^{\star}$ in $R=K[x, y, z] /(F)$, where $F$ is an irreducible homogeneous polynomial of degree $\delta$ such that $R$ is normal. We deal first with the case $a=2$ and consider also the behavior for small degrees and small prime numbers $p$. The computation of $\left(x^{2}, y^{2}, z^{2}\right)^{\star}$ is in fact a question of whether $x y z \in\left(x^{2}, y^{2}, z^{2}\right)^{\star}$ holds or not.

If the degree of $F$ is one, then we may write $x=a y+b z$ and hence $x y z=$ $a y^{2} z+b y z^{2} \in\left(x^{2}, y^{2}, z^{2}\right)$, so $x y z$ belongs to the ideal itself. Suppose that the degree of $F$ is two; hence $F$ defines a quadric. Suppose first that at least one of the mixed monomials $x y, x z, y z$ occurs in $F$, say $x y$. Then the multiple $z F$ shows again that $x y z \in\left(x^{2}, y^{2}, z^{2}\right)$. If however $F=a x^{2}+b y^{2}+c z^{2}$, then 
$K[x, y, z] /\left(F, x^{2}, y^{2}, z^{2}\right)=K[x, y, z] /\left(x^{2}, y^{2}, z^{2}\right)$ and $x y z \notin\left(x^{2}, y^{2}, z^{2}\right)$. If $F$ is irreducible $(a, b, c \neq 0)$, then Spec $R$ is a normal cone over the projective line; hence it is $F$-regular and $x y z \notin\left(x^{2}, y^{2}, z^{2}\right)^{\star}$. If $F$ is the product of two linear forms, then $x y z \in\left(x^{2}, y^{2}, z^{2}\right)^{\star}$, since this (even $\in\left(x^{2}, y^{2}, z^{2}\right)$ ) is true on the two planes and the containment to the solid closure may be checked on the components.

Now suppose that $F \in K[x, y, z]$ has degree 3 and defines an elliptic curve $Y$. If the coefficient of $F$ in $x y z$ is not zero, then of course $x y z \in\left(x^{2}, y^{2}, z^{2}\right)$. Thus we may write $F=S x^{2}+T y^{2}+U z^{2}$, so that $(S, T, U)$ is a homogeneous relation for $\left(x^{2}, y^{2}, z^{2}\right)$ of total degree 3 . If this relation is primary, i.e., $V(S, T, U)=V\left(R_{+}\right)$, then we have a short exact sequence

$$
0 \longrightarrow \varnothing_{Y} \longrightarrow \mathcal{R}(3) \longrightarrow \varnothing_{Y} \longrightarrow 0
$$

and therefore $\mathcal{R}$ is semistable on the elliptic curve $Y$. Hence $x y z \in\left(x^{2}, y^{2}, z^{2}\right)^{\star}$.

If however the relation $(S, T, U)$ is not primary, e.g., for $F=x^{3}+y^{3}+(x+a y) z^{2}$, then we have a decomposition $\mathcal{R}(3)=\varnothing(P) \oplus \varnothing(-P)$ ( $P$ a point $)$ and $x y z \notin$ $\left(x^{2}, y^{2}, z^{2}\right)^{\star}$, since $H^{1}(Y, \mathcal{R}(3))=H^{1}(Y, \varnothing(-P))$ and by Proposition 3.3(ii).

We now examine the case where the equation of the curve is given by a polynomial of degree 4. Note that Proposition 6.2 gives the semistability only for $\delta \geq 5$.

Lemma 7.1. Let $F \in K[x, y, z]$ denote an irreducible homogeneous polynomial of degree 4 and suppose that it defines a smooth projective curve $Y$. Let $\mathcal{R}(m)$ denote the sheaf of relations on $Y$ for the elements $x^{2}, y^{2}, z^{2}$. Then $\mathcal{R}(m)$ is semistable.

Proof. Suppose that $\mathcal{L} \rightarrow \mathcal{R}(3)$ is nontrivial with $\operatorname{deg}(\mathcal{L})>0$. Then $\mathcal{L} \otimes \varnothing_{Y}(1) \rightarrow$ $\mathcal{R}(4)$ is nontrivial and $\operatorname{deg}(\mathcal{L}(1)) \geq 5$; hence $\mathcal{L}(1)$ has global sections $\neq 0$ due to the theorem of Riemann-Roch. It is then enough to show that every nontrivial global section $\varnothing_{Y} \rightarrow \mathcal{R}(4)$ has at most four zeros (counted with multiplicities). Such a section is given by a relation $S=\left(S_{1}, S_{2}, S_{3}\right)$, where the $S_{i}$ are homogeneous polynomials of degree 2 . Since $T=S_{1} x^{2}+S_{2} y^{2}+S_{3} z^{2}=0$ on the curve, we have $T=\lambda F$ in $K[x, y, z], \lambda \in K$.

The zeros of the section $S$ are the common zeros of $\left(S_{1}, S_{2}, S_{3}\right)$. If two of the $S_{1}, S_{2}, S_{3}$ have no common divisor, say $S_{1}$ and $S_{2}$, then $V_{+}\left(S_{1}\right) \cap V_{+}\left(S_{2}\right)$ consists of four points (counted with multiplicities).

So suppose that $S_{1}, S_{2}, S_{3}$ have together one common linear factor $P$. Then we may write $P\left(Q_{1} x^{2}+Q_{2} y^{2}+Q_{3} z^{2}\right)=\lambda F$ in $K[x, y, z]$. For $\lambda \neq 0$ the polynomial $F$ would be reducible, which is excluded. Hence $\lambda=0$. Here $P=0$ would imply that we are dealing with the zero relation; hence $\left(Q_{1}, Q_{2}, Q_{3}\right)$ is a global relation of degree 3. But these are all trivial due to Lemma 6.1; hence this case is not possible.

So suppose that the $S_{i}$ have pairwise one common linear factor. Then the common zeros of $S_{1}, S_{2}, S_{3}$ are the three intersection points of the triangle.

Remark 7.2. The global sections of the sheaf of relations $\mathcal{R}\left(x^{2}, y^{2}, z^{2}\right)(4)$ on a curve of degree 4 depend heavily on the curve equation $F=0$. We always have the standard relations and their linear combinations, but every way of writing $F \in\left(x^{2}, y^{2}, z^{2}\right)$ also yields a relation.

Corollary 7.3. Let $K$ denote an algebraically closed field of characteristic zero. Let $F \in K[x, y, z]$ denote an irreducible homogeneous polynomial of degree $\delta=4$ and suppose that $R=K[x, y, z] /(F)$ is normal. Then $x y z \in\left(x^{2}, y^{2}, z^{2}\right)^{\star}$.

Proof. Lemma 7.1 shows that the sheaf of relations is semistable; hence the numerical criterion Theorem 1.3 (iii) shows that $R_{\geq 3} \subseteq\left(x^{2}, y^{2}, z^{2}\right)^{\star}$. 
Example 7.4. We have a closer look at the Fermat quartic $F=x^{4}+y^{4}+z^{4}$, $\operatorname{char}(K) \neq 2$. We consider the relation for $x^{2}, y^{2}, z^{2}$ of total degree 4 given by $\left(-y^{2}-z^{2}, x^{2}+\sqrt{2} i z^{2}, x^{2}-\sqrt{2} i y^{2}\right)=\left(-y^{2}, x^{2}, 0\right)+\left(-z^{2}, 0, x^{2}\right)+\sqrt{2} i\left(0, z^{2},-y^{2}\right)$. For $z=0$ this relation has no zero on the curve. For $z \neq 0$ we find the four zeros

$$
z=1, \quad y=+/-i, \quad x=+/-\sqrt[4]{2} \sqrt{-i}
$$

and no more. Denote them by $P_{1}, P_{2}, P_{3}, P_{4}$ and let $\Sigma=P_{1}+P_{2}+P_{3}+P_{4}$ be their Weil divisor. The relation $\emptyset_{Y} \rightarrow \mathcal{R}(4)$ then factors through $\emptyset_{Y} \rightarrow \emptyset_{Y}(\Sigma) \rightarrow \mathcal{R}(4)$ and we get an invertible subsheaf of $\mathcal{R}(4)$ without zero, hence a subbundle. This yields a short exact sequence

$$
0 \longrightarrow \varnothing_{Y}(\Sigma) \otimes \varnothing_{Y}(-1) \longrightarrow \mathcal{R}(3) \longrightarrow \varnothing_{Y}(-\Sigma) \otimes \varnothing_{Y}(1) \longrightarrow 0 .
$$

The degree on the left and on the right is 0 . From this it follows not only that $\mathcal{R}(m)$ is semistable, but also that it is strongly semistable by Lemma 3.2. Hence $x y z \in\left(x^{2}, y^{2}, z^{2}\right)^{\star}$ holds on $x^{4}+y^{4}+z^{4}=0$ in positive characteristic $p \geq 3$ also.

Remark 7.5. Note the difference between the sequence in degree three and in the Fermat example of degree four. Both show that $\mathcal{R}$ is strongly semistable but not stable. The first sequence shows at once that $x y z \in\left(x^{2}, y^{2}, z^{2}\right)^{+}$, which is not clear at all in Example 7.4.

Example 7.6. Let $F=z x^{3}+x y^{3}+y z^{3}=0$. If we consider this equation as a relation for $x^{2}, y^{2}, z^{2}$, then this has exactly three zeros (as in the last part of the proof of Lemma [7.1). We get a sequence

$$
0 \longrightarrow \varnothing\left(P_{1}+P_{2}+P_{3}-H\right) \longrightarrow \mathcal{R}(3) \longrightarrow \varnothing\left(H-P_{1}-P_{2}-P_{3}\right) \longrightarrow 0 .
$$

Is $\mathcal{R}(3)$ stable? Is it strongly semistable in positive characteristic?

We look now at the situation of $\operatorname{deg}(F)=\delta \geq 5$. We know that $x y z \in$ $\left(x^{2}, y^{2}, z^{2}\right)^{\star}$ holds in $R=K[x, y, z] /(F)$ for $\operatorname{deg}(F)=\delta \geq 5$ in characteristic zero due to Corollary 6.3. This is in general not true in positive characteristic. It is not even clear whether or not $x y z \in\left(x^{2}, y^{2}, z^{2}\right)^{\star}$ holds on $x^{\delta}+y^{\delta}+z^{\delta}, \delta \geq 5$, for infinitely many or almost all prime characteristics.

Example 7.7. We consider the ideal $\left(x^{2}, y^{2}, z^{2}\right)$ on the curve given by $x^{7}+y^{7}+z^{7}=$ 0 for characteristic $p=3$. The curve equation gives at once a global relation for the elements $x^{6}, y^{6}, z^{6}$ (the third power of $x^{2}, y^{2}, z^{2}$ ) of total degree 7 . Therefore we have the short exact sequence $0 \rightarrow \varnothing_{Y} \rightarrow \mathcal{R}\left(x^{6}, y^{6}, z^{6}\right)(7) \rightarrow \emptyset_{Y}(-4) \rightarrow 0$ showing that $\mathcal{R}\left(x^{2}, y^{2}, z^{2}\right)$ is not strongly semistable (see also Proposition 6.9). To decide whether $x y z \in\left(x^{2}, y^{2}, z^{2}\right)^{\star}$ holds, we have to look at

$$
0 \longrightarrow \varnothing_{Y}(2) \longrightarrow \mathcal{R}\left(x^{6}, y^{6}, z^{6}\right)(9) \longrightarrow \varnothing_{Y}(-2) \longrightarrow 0 .
$$

The element $(x y z)^{3}$ yields the cohomology class

$$
\left(\frac{(x y z)^{3}}{x^{6}},-\frac{(x y z)^{3}}{y^{6}}, 0\right) \in H^{1}\left(Y, \mathcal{R}\left(x^{6}, y^{6}, z^{6}\right)(9)\right),
$$

which maps to $\frac{(x y z)^{3} z}{x^{6} y^{6}}=\frac{z^{4}}{x^{3} y^{3}} \in H^{1}\left(Y, \varnothing_{Y}(-2)\right)$ by Remark 4.8 This class is not zero. Therefore the (dual) extension $0 \rightarrow \varnothing_{Y} \rightarrow \mathcal{G} \rightarrow \varnothing_{Y}(2) \rightarrow 0$ given by this class is not trivial. From this and from $\operatorname{deg}(\mathcal{G})=14>\frac{2}{3}(15-1)$ we see that $\mathcal{G}$ is ample. Hence $z^{4} \notin\left(x^{3}, y^{3}\right)^{\star}$ and then also $x y z \notin\left(x^{2}, y^{2}, z^{2}\right)^{\star}$. 
We now look at $\left(x^{3}, y^{3}, z^{3}\right)^{\star}$ in order to extend the results from the last section for the Fermat equations $x^{\delta}+y^{\delta}+z^{\delta}=0$ of low degrees. For $\delta=1,2$ we have $\left(x^{3}, y^{3}, z^{3}\right)^{\star}=\left(x^{3}, y^{3}, z^{3}\right)$. For $\delta=3$ we have $\left(x^{3}, y^{3}, z^{3}\right)^{\star}=\left(x^{3}, y^{3}\right)^{\star}=\left(x^{3}, y^{3}\right)+$ $R_{\geq 6}$.

Example 7.8. For $x^{4}+y^{4}+z^{4}=0$ we have the short exact sequence $0 \rightarrow \emptyset_{Y} \rightarrow$ $\mathcal{R}(4) \rightarrow \emptyset_{Y}(-1) \rightarrow 0$ given by the relation $(x, y, z)$. Therefore the sheaf of relations $\mathcal{R}(m)$ for $x^{3}, y^{3}, z^{3}$ is not semistable on the Fermat quartic. The sheaf $\mathcal{R}(5)$ is also not ample. It is however the extension of two invertible sheaves of degree $\geq 0$; hence it follows by Proposition 3.3 that $R_{\geq 5} \subset\left(x^{3}, y^{3}, z^{3}\right)^{\star}$ and also that $R_{\geq 5} \subset\left(x^{3}, y^{3}, z^{3}\right)^{+}$in positive characteristic.

Example 7.9. For $x^{5}+y^{5}+z^{5}=0$ we have the short exact sequence $0 \rightarrow \emptyset_{Y} \rightarrow$ $\mathcal{R}(5) \rightarrow \emptyset_{Y}(1) \rightarrow 0$ given by the relation $\left(x^{2}, y^{2}, z^{2}\right)$. This extension is not trivial, since there does not exist a nontrivial mapping $\emptyset_{Y} \rightarrow \mathcal{R}(4)$. Therefore the sheaf $\mathcal{R}(5)$ is ample in characteristic zero and hence also for $p \gg 0$. To obtain a bound for the prime number, we use the criterion of Mumford-Hartshorne. Suppose that there exists an invertible quotient sheaf $\mathcal{R}(5) \rightarrow \mathcal{L} \rightarrow 0$ of $\operatorname{deg}(\mathcal{L}) \leq 0$. The exact sequence shows at once that $\mathcal{L}=\emptyset_{Y}$ and hence that it would split. Hence it follows that $\left(x^{3}, y^{3}, z^{3}\right)^{\star}=\left(x^{3}, y^{3}, z^{3}\right)+R_{\geq 5}$ for $p>2$.

Proposition 7.10. Let $R=K[x, y, z] /\left(x^{\delta}+y^{\delta}+z^{\delta}\right)$ and let $\mathcal{R}(m)$ denote the sheaf of relations for the elements $x^{3}, y^{3}, z^{3}$ of total degree $m$. Then $\mathcal{R}(5)$ is an ample sheaf for $\delta \geq 5$ and $p \geq \delta-3$ (or in characteristic zero).

Proof. The result follows for $\delta \geq 8$ from Corollary 6.4 and for $\delta=5$ from Example 7.9. So suppose that $\delta=6$ or $=7$. We apply the criterion of Hartshorne-Mumford; thus we assume that the invertible quotient sheaf $\mathcal{R}(5) \rightarrow \mathcal{L} \rightarrow 0$ has degree $\leq 0$. Then there exists an invertible subsheaf of $\mathcal{R}(5)$ of degree $\geq \delta$. Then $\mathcal{R}(6)$ contains an invertible subsheaf $\mathcal{M}$ of degree $\geq 2 \delta$ and $\mathcal{R}(7)$ contains an invertible subsheaf $\mathcal{M}$ of degree $\geq 3 \delta$. From Riemann-Roch we see that these subsheaves of $\mathcal{R}(\delta)$ have nontrivial global sections.

Let $\delta=6$ and consider a global relation of total degree 6 . It is given by $S_{1} x^{3}+$ $S_{2} y^{3}+S_{3} z^{3}=\lambda F$ on the curve. The $S_{i}$ do not have a common divisor, for then $\lambda=0$ and the relation would be a multiple of a relation of $\mathcal{R}(k), k \leq 5$, but these are zero. This implies that the polynomials $S_{1}$ and $S_{2}$ (say) have at most a linear form in common. Then the number of zeros is bounded by $6+4$ or by 9 ; hence it is $\leq 12$.

Consider now $\delta=7$ and let $S_{1} x^{3}+S_{2} y^{3}+S_{3} z^{3}$ denote a relation on the curve of total degree 7 ; hence $\operatorname{deg}\left(S_{i}\right)=4$. The three polynomials together have a common divisor of degree at most 1 , since $\Gamma(Y, \mathcal{R}(5))=0$. So two of the polynomials, say $S_{1}$ and $S_{2}$, have a common divisor $C$ of degree $t$ at most 2 . If $t=2$, then the number of zeros is bounded by $2 \cdot 7+4$; if $t=1$, then it is bounded by $7+9$; and for $t=0$ it is bounded by 16 , so in any case $\leq 21$.

\section{REFERENCES}

1. H. Brenner, Tight closure and projective bundles, J. Algebra 265 (2003), 45-78. MR 1984899 (2004h:13008)

2. H. Brenner, The slope of vector bundles and applications to tight closure problems, Trans. Amer. Math. Soc. 356 (1) (2004), 371-392. MR2020037

3. D. Gieseker, p-ample bundles and their Chern classes, Nagoya Math. J. 43 (1971), 91-116. MR0296078|(45:5139) 
4. A. Grothendieck and J. Dieudonné, Eléments de géométrie algébrique II. Inst. Hautes Études Sci. Publ. Math. 8 (1961). MR0217084 (36:177b)

5. N. Hara, A characterization of rational singularities in terms of injectivity of Frobenius maps, Amer. J. of Math. 120, 5 (1998), 981-996. MR1646049 (99h:13005)

6. G. Harder, M. S. Narasimhan, On the cohomology groups of moduli spaces of vector bundles on curves, Math. Ann. 212 (1975), 215-248. MR0364254 (51:509)

7. R. Hartshorne, Ample vector bundles, Publ. Math. I.H.E.S. 29 (1966), 63-94. MR0193092 $(33: 1313)$

8. R. Hartshorne, Algebraic Geometry, Springer-Verlag, New York, 1977. MR0463157(57:3116)

9. M. Hochster, Solid closure, Contemp. Math. 159 (1994), 103-172. MR.1266182 (95a:13011)

10. M. Hochster, C. Huneke, Tight closure, invariant theory, and the Briançon-Skoda theorem, J. Amer. Math. Soc. 3 (1990), 31-116. MR.1017784 (91g:13010)

11. C. Huneke, Tight Closure and Its Applications, AMS, 1996. MR.1377268 (96m:13001)

12. C. Huneke, Tight Closure, Parameter Ideals, and Geometry, in Six Lectures on Commutative Algebra, Birkhäuser, Basel, 1998. MF 1648666 (99j:13001)

13. C. Huneke, K. Smith, Tight closure and the Kodaira vanishing theorem, J. Reine Angew. Math. 484 (1997), 127-152. MR1437301 (98e:13007)

14. D. Huybrechts, M. Lehn, The Geometry of Moduli Spaces of Sheaves, Viehweg, Braunschweig, 1997. MR1450870 (98g:14012)

15. R. Lazarsfeld, Positivity in Algebraic Geometry (Preliminary Draft), 2001.

16. G. J. Leuschke, Appendix: Some examples in tight closure, Trends in Commutative Algebra, MSRI publications, 51, 2004.

17. L. Migliorini, Some observations on cohomologically $p$-ample bundles, Annali di Matematica pura ed applicata (IV), Vol CLXIV, 89-102, (1993). MR.1243950 (94k:14012)

18. Y. Miyaoka, The Chern class and Kodaira dimension of a minimal variety, in Algebraic Geometry, Sendai 1985, Adv. Stud. Pure Math. 10, 1987, 449-476. MR0946247|(89k:14022)

19. M. Katzman, Gröbner bases and the Frobenius map, preprint, http://www.sheffield. ac.uk/katzman/Articles/Publications.html.

20. A. Singh, A computation of tight closure in diagonal hypersurfaces, J. Algebra 203, No. 2 (1998), 579-589. MR 1622811|(2000d:13007)

21. K. E. Smith, Tight closure in graded rings, J. Math. Kyoto Univ 37, No. 1 (1997), 35-53. MR.1447362 (98e:13009)

22. K. E. Smith, An introduction to tight closure, in Geometric and combinatorial aspects of commutative algebra, Lect. Notes Pure Appl. Math. 217 (2001), 353-377. MR1824242 (2002e:13011)

23. S. Sullivant, Tight closure of monomial ideals in Fermat rings, preprint, 2002.

Department of Pure Mathematics, University of Sheffield, Hicks Building, HounsField Road, ShefField S3 7RH, United Kingdom

E-mail address: H.Brenner@sheffield.ac.uk 